\title{
ANALYTISCHER UND SYNTHETISCHER GENITIV IM DEUTSCHEN - EIN DIACHRONER VERGLEICH ${ }^{1}$
}

\section{Einleitung}

Die deutsche Sprache gehört zu der Gruppe germanischer Sprachen, die neben dem relativ häufig gebrauchten analytischen Genitiv auch die ältere Form des synthetischen Genitivs beibehalten haben (vgl. das Isländische, Schwedische usw.). Der synthetische Genitiv ist eine attributive Nominalgruppe, in der das Verhältnis zum Bezugsnomen durch Flexionsformen angezeigt wird (daz buoch der wîsheit /Meister Eckhart/), wohingegen mit dem analytischen Genitiv eine attributive Präpositionalgruppe gemeint ist, die die Präposition von enthält und einen attributiven synthetischen Genitiv ersetzen kann. Die Präposition von hat eine vergleichbare Funktion wie die oben erwähnte Flexionsform (eine Vielheit von Personen /Matthias Scheeben/). Beide Genitivformen sind sowohl in den ältesten Entwicklungsstufen des Deutschen wie auch in jüngeren Perioden Konkurrenzformen. Das Thema des vorliegenden Beitrages ist das Verhältnis zwischen dem Gebrauch beider Formen und die Intensität, mit der der analytische Genitiv den synthetischen ersetzt. Es ist ein diachroner Vergleich und beide Genitivformen sind so anhand Belege aus allen sprachgeschichtlichen Perioden der deutschen Sprache untersucht, verglichen und statistisch bearbeitet worden.

Die theoretische Grundlage der Untersuchung bilden die Erkenntnisse der natürlichen Syntax bzw. der sogenannten slowenischen Schule der natürlichen Syntax. Hinsichtlich dieser Erkenntnisse ist untersucht worden, ob der analytische Genitiv vom diachronen Standpunkt aus als verstärkte Konstruktion bewertet werden kann.

1 Der Beitrag ist eine Zusammenfassung der Magisterarbeit, die am 14.11.1997 an der Pädagogischen Fakultät in Maribor vor der Kommission Prof.Dr. Janez Orešnik (Mentor), Prof.Dr. Karmen Teržan Kopecky (Komentorin) und Prof.Dr. Mirko Križman verteidigt wurde. Die Kapitel über beide Genitivformen in den Grammatiken des Gegenwartsdeutschen und über die Verbreitung des analytischen Genitivs in slawischen und in germanischen Sprachen außerhalb des Deutschen werden hier nicht zusammengefaßt. 


\section{Theoretische Ausgangspunkte und Vorhersagen}

Die slowenische Schule $^{2}$ der natürlichen Syntax unterscheidet zwischen verstärkten grammatischen Konstruktionen und geschwächten grammatischen Konstruktionen. Beim Vergleich der Konstruktionen synthetischer vs. analytischer Genitiv wird der Variante analytischer Genitiv der Status einer verstärkten Konstruktion eingeräumt. Die Präpositionalgruppe mit von (analytischer Genitiv) weist nämlich folgende Eigenschaften verstärkter Konstruktionen auf:

1. Der analytische Genitiv ist formal umständlicher als die entsprechende geschwächte Variante (der synthetische Genitiv). Das Verhältnis zum Bezugsnomen wird im Falle des analytischen Genitivs durch die Einfügung eines zusätzlichen Lexems realisiert und ein anderer Kasus wird gebraucht (Dativ):

\section{das alte Haus von meinem Vater vs, das alte Haus meines Vaters}

2. Der analytische Genitiv weist eine semantisch spezialisiertere Untermenge von Bedeutungen als der synthetische Genitiv auf, daher kann es nicht alle Arten des synthetischen Genitivs ersetzen. Den Grammatiken der modernen deutschen Sprache entnehmen wir, daß der analytische Genitiv verschiedene Arten des synthetischen Genitivs ersetzen kann wie Genitivus partitivus, Genitivus possessivus, Genitivus subjectivus, Genitivus objectivus, Genitivus qualitatis, Genitiv der Steigerung und die Nominalgruppe, in der die Verbindung von zwei oder mehreren Genitiven vorkommt. Grundsätzlich ist also in fast allen Arten von Genitivverbindungen die Umschreibung mit der Präpositionalgruppe möglich, aber ob sie wirklich eintritt, hängt von verschiedenen Faktoren ab. Verschiedene Einschränkungen sind z.B. das Erscheinen eines zusätzlichen Attributs oder des bestimmten Artikels - in solchen Fällen tritt der analytische Genitiv nicht ein. ${ }^{3}$ Der stilistische Unterschied zwischen dem analytischen und synthetischen Genitiv wird in der Fachliteratur oft erwähnt; der Variante analytischer Genitiv wird umgangssprachlicher Charakter zugeschrieben. Die Tendenz, allmählich alle Arten des Genitivs durch die analytische Form zu ersetzen, zeigt sich gerade in dem umgangssprachlichen Gebrauch der Ersatzstruktur. ${ }^{4}$

3. Dem Sprecher bereitet das Produzieren einer analytischen Genitivform mit einem zusätzlichen Lexem größere Schwierigkeiten als das Produzieren einer synthetischen Genitivform.

2 Näheres dazu in Orešnik (1990, 1995), Teržan (1990), Teržan Kopecky (1993) und Petrič (1990).

3 Auf genauere Beschreibungen möchten wir an dieser Stelle verzichten. Näheres ist im Abschnitt 5 zu finden.

4 Vgl. dazu Boretzky (1977:160): "In dt. Dialekten ist die alte Form des Gen. fast ganz durch Präpositionalausdrücke, vor allem mit von, verdrängt worden, so daß anstelle des alten Paradigmas der Wagen, des Wagens, dem Wagen, den Wagen nun gilt der Wagen, vom Wagen, dem Wagen, den Wagen. Damit hat sich, wohlgemerkt, das alte Kasussystem noch nicht aufgelöst, sondern in das Paradigma ist anstelle einer synthetischen eine analytische Form aufgenommen worden." 
4. Dem Hörer erleichtert die Präposition von als sprachliches Zeichen das Verständnis der analytischen Konstruktion.

Für den synthetischen Genitiv als eine geschwächte Konstruktion gilt das Umgekehrte; die Konstruktion ist formal weniger umständlicher, sie ist semantisch weniger spezialisiert, der Sprecher kann sie leichter bilden und für den Hörer ist sie schwieriger zu dekodieren.

Das hier beschriebene Modell der natürlichen Syntax konzentriert sich auf den syntaktischen Wandel. Aus der Sicht der geschichtlichen Entwicklung einer verstärkten und einer geschwächten Konstruktion wurde folgende Grundannahme ausgearbeitet (Orešnik et al. 1990, Orešnik 1995): am Anfang ${ }^{5}$ behaupten sich verstärkte Konstruktionen vorzugsweise unter relativ komplizierten grammatischen Verhältnissen und verbreiten sich später möglicherweise auch unter weniger komplizierten grammatischen Verhältnissen. Geschwächte Konstruktionen behaupten sich dagegen am Anfang vorzugsweise unter relativ einfachen grammatischen Verhältnissen und verbreiten sich später möglicherweise auch unter weniger einfachen grammatischen Verhältnissen.

Der analytische Genitiv hat sich in der Geschichte der deutschen Sprache relativ spät etabliert, sein Gebrauch ist ein Zeichen der Entwicklungstendenzen in der deutschen Sprache, die darauf hinweisen, daß die einst vorwiegend synthetische Sprache schrittweise in eine analytische übergeht. Auch andere analytische Konstruktionen haben sich relativ spät durchgesetzt: z.B. die Verbalkategorien Perfekt, Passiv, Futur oder die Entwicklung des Artikels, der die Kasusendungen ersetzte bzw. ein zusätzliches Signal für die im Satz auftretenden syntaktischen Verhältnisse darstellt. Gemäß der Grundannahme war zu erwarten, daß der analytische Genitiv in den ersten Entwicklungsstufen, in denen die Variante zunächst als eine Ersatzkonstruktion erscheint, unter relativ komplizierten grammatischen Verhältnissen vorkommt und sich in späteren Jahrhunderten unter weniger komplizierten Verhältnissen verbreitet. Im Falle des synthetischen Genitivs wird für ältere Entwicklungsstufen das Vorkommen unter relativ einfachen grammatischen Verhältnissen und die spätere Durchsetzung unter weniger einfachen vermutet.

Bei der Frage, was in der Sprache kompliziert ist und was einfach, folgt die slowenische Schule der natürlichen Syntax der Markiertheitstheorie und akzeptiert in den meisten Fällen die diesbezüglichen Vorschläge von Mayerthaler (1981). So ist auch in Anlehnung an den erwähnten theoretischen Hintergrund bei der Analyse des synthetischen und analytischen Genitivs davon auszugehen, daß Elemente mit hohem Markiertheitsgrad am natürlichsten mit formal komplizierten Ausdruckselementen kodiert werden, z.B. analytischen Konstruktionen.

Markierte und unmarkierte Werte sind für jeden beobachteten grammatischen Parameter bestimmt worden. Markierte Werte wären:

5 Das bedeutet in dem Stadium, in welchem eine Konstruktion nur als eine syntaktische Variante eines anderen Ausdrucks zu betrachten ist und noch nicht grammatikalisiert ist. 
- ein synthetischer Genitiv, erweitert durch Attribute oder ein durch einen weiteren Genitiv

(1) der ganz Verstand christlicher Gnad, Freiheit, Glaubens /Luther/,

- ein analytischer Genitiv, erweitert durch Attribute oder ein durch einen weiteren Dativ

(2) der Glaubensfaß von den beiden Prozessionen und Produktionen /Scheeben/,

- ein Kern der Nominalgruppe, erweitert durch Attribute oder ein durch eine weitere Nominalphrase

(3) der begehrte Grund und Ursach euer Hoffnung /Luther/

(4) ein Ausfluß und tatsächliches Abbild von der Einheit/Scheeben/,

- ein synthetischer oder analytischer Genitiv mit einem Komparativ oder Superlativ (=diese sind markierter als Positive), das bezieht sich auch auf die Bezugsgröße

(5) die Enthülung des volleren und klareren Begriffes /Scheeben/, die hoechsten krefte der sêle, der nature /Eckhart/,

- ein synthetischer oder analytischer Genitiv ohne pronominale Elemente, denn diese betrachten wir als unmarkierte grammatische Verhältnisse bzw. grammatische Umgebung, das bezieht sich auch auf die Bezugsgröße

(6) und er blîbet in vride sînes herzen unbeweget /Eckhart/,

- ein synthetischer oder analytischer Genitiv als Akkusativobjekt im Satz, verglichen mit einem Subjekt, denn ein Akkusativobjekt ist markierter als ein Subjekt, das bezieht sich auch auf die Bezugsgröße

(7) Er hat doch auch anderseits einen so tiefen Kern von Wahrheit/Scheeben/,

- ein synthetischer oder analytischer Genitiv im Rhema des Satzes, denn das Rhema eines Satzes ist eine markiertere grammatische Umgebung im Vergleich zum Thema, das bezieht sich auch auf die Bezugsgröße

(8) Sie haben einen Schein der Frummkeit /Luther/,

- eine Nominalgruppe mit einer größeren Anzahl von flektierbaren Wörtern ist markierter, denn je höher die Zahl der flektierbaren Wörter, desto höher der Markiertheitsgrad; das betrifft auch die Bezugsgröße.

Auf Grund des o.e. wurden folgende Vorhersagen gebildet:

- der analytische Genitiv ist häufiger erweitert durch Attribute (=eine höhere Zahl der Attribute) als der synthetische Genitiv; das bezieht sich auch auf die Bezugsgröße,

- in den nominalen Attributen treten Komparative und Superlative häufiger in den Belegen des analytischen Genitivs auf als in den Belegen des synthetischen Genitivs,

- der analytische Genitiv kommt häufiger in Objekt vor als der synthetische Genitiv, 
- der analytische Genitiv kommt häufiger im Rhema des Satzes vor als der synthetische Genitiv,

- der analytische Genitiv weist eine höhere Zahl der flektierbaren Wörter als der synthetische Genitiv auf.

Die grammatischen Parameter des analytischen Genitivs sollten gemäß der Grundannahme markiertere Werte aufweisen, da ja unsere Grundannahme besagt, daß die Vorkommenswahrscheinlichkeit des markierten Wertes von einem grammatischen Parameter in einer verstärkten Konstruktion größer ist als die Vorkommenswahrscheinlichkeit des markierten Wertes von demselben Parameter in der entsprechenden geschwächten Konstruktion. Der Unterschied sollte sich als statistisch signifikant erweisen.

Laut Grundannahme ist es möglich, daß sich die grammatische Umgebung beider Genitivformen im Laufe der geschichtlichen Entwicklung des Deutschen verändert hat. Die folgenden Vorhersagen beziehen sich auf den syntaktischen Wandel:

- der analytische Genitiv kommt in den ersten Jahrhunderten, in denen er neben dem synthetischen Genitiv als syntaktische Variante auftritt (Althochdeutsch, Mittelhochdeutsch), unter relativ markierten grammatischen Verhältnissen vor. In den folgenden Jahrhunderten erscheint der analytische Genitiv möglicherweise auch unter weniger markierten grammatischen Verhältnissen, die an der Abnahme markierter Werte einzelner Parameter erkennbar werden,

- der synthetische Genitiv kommt umgekehrt in den ältesten sprachgeschichtlichen Perioden in einer relativ einfachen grammatischen Umgebung vor. In späteren Perioden erscheint die Konstruktion laut Annahme möglicherweise auch unter weniger einfachen Verhältnissen.

Neben den Unterschieden, die die grammatische Umgebung betreffen, sind in den Stichproben mit den beiden Konstruktionsvarianten (d.h. einer verstärkten und einer geschwächten Konstruktion) auch Unterschiede zu erwarten, die ihre Vorkommenshäufigkeit oder Frequenz der Belege betreffen. Eine natürliche (weniger markierte) sprachliche Kategorie wird häufiger verwendet als eine weniger natürliche. Demgemäß wäre es zu erwarten, daß der synthetische Genitiv als die geschwächte Variante in Texten aus allen sprachgeschichtlichen Perioden häufiger vorkommt.

Wie schon erwähnt, sind Belege aus Texten aus allen sprachgeschichtlichen Perioden der deutschen Sprache untersucht worden. Bei der Auswahl der Texte mußte berücksichtigt werden, daß die Texte lang genug sind, damit genügend Belege für die intendierte statistische Analyse vorliegen und daß es sich um Texte einer Textsorte handelt. Als geeignete Texte wurden natürliche Gesprächsabläufe betrachtet. Solche Gesprächssituationen sind aber in den Texten aus den ältesten sprachgeschichtlichen Perioden nur selten zu finden. Als Datenbasis mußten daher folgende theologische Schriften dienen:

- Althochdeutsch: Der althochdeutsche Isidor (3., 4. Kapitel), 
- Mittelhochdeutsch: Meister Eckhart. Daz buoch der goetlîchen Troestunge,

- Frühneuhochdeutsch: Martin Luther. Von der Freiheit eines Christenmenschen, Eine treue Vermahnung zu allen Christen, sich zu hüten vor Aufruhr und Empörung, An die Ratsherrn aller Städte deutsches Lands, daß sie die christliche Schulen aufrichten und halten sollen,

- Neuhochdeutsch, Barock: Abraham a Sancta Clara. Neuerwöhlte ParadeysBlum, Aller Freud/und Fried ist Ursach Maria,

- Neuhochdeutsch, 19.Jhr.: Matthias Scheeben. Die Mysterien des Christentums.

\section{Historische Entwicklung beider Genitivkonstruktionen}

Im Althochdeutschen finden wir erste Belege des analytischen Genitivs (vgl. Dal 1966, 1977, Behaghel 1923, Leiss 1991); die Präpositionalgruppe mit von ersetzte zu der Zeit zuerst den Genitivus partitivus, später in der althochdeutschen Periode auch andere Genitivarten (sunu fona fater /Isidor/, ther keisor fona Rumu /Otrfrid von Weissenburg/). Für die Zeit des Mittelhochdeutschen (vgl. Dal 1966, Behaghel 1923, Leiss 1991) werden keine wesentlichen Veränderungen im Gebrauch beider Genitivformen beobachtet. Einzelne Beispiele der Ersatzstruktur sind in verschiedenen Texten zu finden (gotes sun von gnâden /Meister Eckhart/), doch die Belege des analytischen Genitivs blieben weiterhin spärlich. Im Frühneuhochdeutschen ist erstmals ein intensiverer Gebrauch der analytischen Konstruktionen festzustellen (darüber u.a. Dal 1966, Behaghel 1923, Admoni 1985, Leiss 1991); zu erwähnen wären z.B. die Verbalkategorien Futur, Passiv, Perfekt. Was den analytischen Genitiv betrifft (einer von denen /Luther/), können wir schon von einer etablierten Konstruktion sprechen und nicht nur von einzelnen Beispielen. Dabei ist der synthetische Genitiv in seinem Gebrauch jedoch nicht dermaßen gefährdet, daß er seinen Platz der analytischen Variante überlassen müßte. Admoni (1985) stellt zum Beispiel fest, daß der Umfang des Gebrauchs des analytischen Genitivs nicht so groß war, um größere Veränderungen in der Sprache zu verursachen. Für das Neuhochdeutsche sind keine größeren Veränderungen im Verhältnis synthetischer vs. analytischer Genitiv festgestellt worden (vgl. Admoni 1985).

Für alle sprachgeschichtlichen Perioden wie auch für das moderne Standarddeutsch wird beobachtet, daß der analytische Genitiv den synthetischen besonders dann ersetzt, (a) wenn die Genitivendung nicht transparent genug ist, (b) wenn es sich um Verbindungen von zwei oder sogar mehreren Genitivattributen handelt oder z.B. (c) wenn im Genitivattribut Nominalphrasen verschiedener Genera vorkommen, wie auch in anderen Situationen. Aber auch das Standarddeutsche erlebte Einflüsse aus der Umgangssprache, in der der analytische Genitiv eigentlich ohne Einschränkungen gebraucht wird (vgl. dazu die Einigkeit von unserm Hause /Grillparzer/, wir sind alle Diener von seiner Exzellenz /Weise/). 


\section{Analyse $e^{6}$}

In den Tabellen sind folgende Abkürzungen gebraucht worden (Parameter A bis I):

A - : erweiterter synthetischer/ analytischer Genitiv, 뜨: nicht-erweiterter synthetischer/ analytischer Genitiv

B - Zahl der Attribute im synthetischen/ analytischen Genitiv

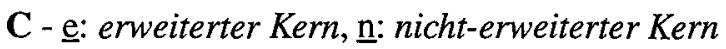

D - Zahl der Attribute im Kern

E - Zahl der Komparative und Superlative im synthetischen/ analytischen Genitiv und im Kern

F - Zahl der Pronomina im synthetischen/ analytischen Genitiv und im Kern

G - ․: synthetischer/ analytischer Genitiv als Akkusativobjekt, $\underline{\mathrm{s}}$ : synthetischer/ analytischer Genitiv als Subjekt

H - ㅁ: synthetischer/ analytischer Genitiv als Rhema, $\underline{\mathrm{t}}$ : synthetischer/ analytischer Genitiv als Thema

I - Zahl der flektierbaren Wörter im synthetischen/ analytischen Genitiv

Tabelle 1: Der althochdeutsche Isidor/synthetischer Genitiv/

\begin{tabular}{|c|c|c|c|c|c|c|c|c|c|c|}
\hline$S$ & Text & A & $\mathrm{B}$ & $\mathrm{C}$ & $\mathrm{D}$ & $\mathrm{E}$ & $\mathrm{F}$ & G & $\mathrm{H}$ & I \\
\hline 252 & Araugit ist in dhes aldin uuizssodes boohhum, & $\mathrm{e}$ & 1 & $\mathrm{n}$ & 0 & 0 & 0 & 1 & $\mathrm{r}$ & 3 \\
\hline 255 & $\begin{array}{l}\text { bidhiu huuanda sie chihordon gotes stimna hluda in } \\
\text { Sinaberge quhedhenda:, }\end{array}$ & $\mathrm{n}$ & 0 & $\mathrm{e}$ & 1 & 0 & 0 & o & $\mathrm{r}$ & 3 \\
\hline 260 & $\begin{array}{l}\text { nalles sie dhrie goda, oh ist in dhesem dhrim heidem } \\
\text { ein namo dhes unchideiliden meghines., }\end{array}$ & $\mathrm{e}$ & 1 & $\mathrm{n}$ & 0 & 0 & 0 & $\mathrm{~s}$ & $\mathrm{r}$ & 3 \\
\hline 264 & $\begin{array}{l}\text { in dhemu eristin deile chuningo boohho sus ist } \\
\text { chiuuisso chiscriban: ... }\end{array}$ & $\mathrm{e}$ & 1 & $\mathrm{e}$ & 1 & 0 & 0 & I & $r$ & 4 \\
\hline 265 & ..."Quhad Dauid, Isais sunu, quhad gomman, ... & $\mathrm{n}$ & 0 & $\mathrm{n}$ & 0 & 0 & 0 & $\mathrm{~s}$ & $\mathrm{t}$ & 2 \\
\hline 266 & $\begin{array}{l}\text {...dhemu izs chibodan uuard umbi Christan Iacobes } \\
\text { gotes, ... }\end{array}$ & $\mathrm{n}$ & 0 & $\mathrm{n}$ & 0 & 0 & 0 & 1 & $\mathrm{r}$ & 2 \\
\hline 267 & ...dher erchno sangheri Israhelo: ... & $\mathbf{n}$ & 0 & $\mathrm{e}$ & 1 & 0 & 0 & $\mathrm{~s}$ & $r$ & 3 \\
\hline 267 & ..."Gotes gheist ist sprehhendi dhurah mih, & $\mathrm{n}$ & 0 & n & 0 & 0 & 0 & $\mathrm{~s}$ & $\mathrm{t}$ & 2 \\
\hline 270 & "Israhelo got was mir zuo sprehhendi, ... & $\mathrm{n}$ & 0 & $\mathrm{n}$ & 0 & 0 & 0 & $\mathrm{~s}$ & $\mathrm{t}$ & 2 \\
\hline 271 & $\begin{array}{l}\text {...dher rehtuuisigo manno uualdendeo, (Israhelo } \\
\text { got) ... }\end{array}$ & $\mathrm{n}$ & 0 & $\mathrm{e}$ & 1 & 0 & 0 & $\mathrm{~s}$ & $r$ & 3 \\
\hline 271 & ...strango Israhelo." & $\mathrm{n}$ & 0 & $\mathrm{n}$ & 0 & 0 & 0 & $\mathrm{~s}$ & $\mathrm{r}$ & 2 \\
\hline 274 & $\begin{array}{l}\text { Dhar ir auh quhad: "Gotes gheist ist sprehhendi } \\
\text { dhurah mih", dhar meinida }\end{array}$ & $\mathrm{n}$ & 0 & $\mathrm{n}$ & 0 & 0 & 0 & $s$ & $\mathrm{t}$ & 2 \\
\hline 278 & "Druhtines uuordu sindun himila chifestinode & $\mathrm{n}$ & 0 & $n$ & 0 & 0 & 0 & 1 & $r$ & 2 \\
\hline
\end{tabular}

6 Die Gesamtzahl der analysierten Belege beider Genitivformen betrifft 1552 Belege. Hier sind nur 279 Belege aus verschiedenen Tabellen zu finden. 


\begin{tabular}{|c|c|c|c|c|c|c|c|c|c|c|}
\hline 280 & $\begin{array}{l}\text {...In dhemu druhtines nemin archennemes } \\
\text { chiuuisso fater, }(\ldots) \text {... }\end{array}$ & $\mathrm{n}$ & 0 & $n$ & 0 & 0 & 0 & 1 & $t$ & 2 \\
\hline 281 & $\begin{array}{l}\text {...in sines mundes gheiste instandemes chiwisso } \\
\text { heilegan gheist. }\end{array}$ & $\mathrm{e}$ & 1 & $\mathrm{n}$ & 0 & 0 & 1 & 1 & $t$ & 3 \\
\hline 283 & $\begin{array}{l}\text { In dheseru urchundin ist ziuuare araughit dhera } \\
\text { dhrinissa zala ... }\end{array}$ & $\mathrm{n}$ & 0 & $\mathrm{n}$ & 0 & 0 & 0 & $\mathrm{~s}$ & $\mathrm{r}$ & 2 \\
\hline 291 & $\begin{array}{l}\text { "In dhemu eristin chideda got himil endi aerdha endi } \\
\text { gotes gheist suueiboda oba uuazsserum." }\end{array}$ & $\mathrm{n}$ & 0 & $\mathrm{n}$ & 0 & 0 & 0 & $\mathrm{~s}$ & $\mathrm{t}$ & 2 \\
\hline 292 & $\begin{array}{l}\text { Dhar ist auh ih dhemu gotes nemin fater zi } \\
\text { firstandanne; }\end{array}$ & $\mathrm{n}$ & 0 & $\mathbf{n}$ & 0 & 0 & 0 & 1 & $\mathrm{t}$ & 2 \\
\hline 294 & $\begin{array}{l}\text { "In haubide dhes libelles azs erist ist chiscriban } \\
\text { umbi mih }\end{array}$ & $\mathrm{n}$ & 0 & $\mathrm{n}$ & 0 & 0 & 0 & 1 & $\mathrm{t}$ & 2 \\
\hline 302 & dhurah dhero heideo maneghin ist . & $\mathrm{n}$ & 0 & $\mathrm{n}$ & 0 & 0 & 0 & 1 & $\mathrm{t}$ & 2 \\
\hline 302 & ...dhar chioffonot dhera dhrinissa bauhnunc. & $\mathrm{n}$ & 0 & $\mathrm{n}$ & 0 & 0 & 0 & $\mathrm{~s}$ & I & 2 \\
\hline 303 & $\begin{array}{l}\text { Endi dhoh dhiu huuedheru nu, dhazs ir dhea } \\
\text { einnissa gotes araughida, }\end{array}$ & $\mathrm{n}$ & 0 & $\mathrm{n}$ & 0 & 0 & 0 & 0 & $\mathrm{r}$ & 2 \\
\hline 307 & Dhiu selba maneghiu chinomideo araughit ... & $\mathrm{n}$ & 0 & $\mathrm{e}$ & 1 & 0 & 0 & s & $\mathrm{t}$ & 3 \\
\hline 307 & ...dhazs meghiniga chiruni dhera dhrinissa. & $\mathrm{n}$ & 0 & $\mathrm{e}$ & 1 & 0 & 0 & 0 & $\mathbf{r}$ & 3 \\
\hline 309 & $\begin{array}{l}\text { dhera selbun dhrinissa heilac chiruni Aggeus dher } \\
\text { forasago sus araughida ... }\end{array}$ & $\mathrm{e}$ & 1 & $\mathrm{e}$ & 1 & 0 & 0 & s & $\mathrm{r}$ & 4 \\
\hline 310 & ...in druhtines nemin quhedhendi: & $\mathrm{n}$ & 0 & $\mathrm{n}$ & 0 & 0 & 0 & 1 & $\mathrm{r}$ & 2 \\
\hline 317 & So sama auh araughit ist in Isaies buohhum & $\mathrm{n}$ & 0 & $\mathrm{n}$ & 0 & 0 & 0 & 1 & $\mathrm{r}$ & 2 \\
\hline 318 & $\begin{array}{l}\text {...eochihuueliihhes dhero heideo sundric } \\
\text { undarscheit, }\end{array}$ & $\mathrm{n}$ & 0 & $\mathrm{e}$ & 1 & 0 & 0 & l & $\mathbf{r}$ & 3 \\
\hline 319 & selbemu dhemu gotes sune quhedhendemu: & $\mathrm{n}$ & 0 & $\mathrm{n}$ & 0 & 0 & 0 & $\mathrm{~s}$ & $\mathrm{t}$ & 2 \\
\hline 326 & $\begin{array}{l}\text { endi dher dhritto heit ist selbes druhtines Christes } \\
\text { dhes chisendidin. }\end{array}$ & $\mathrm{n}$ & 0 & $\mathrm{n}$ & 0 & 0 & 0 & 1 & $\mathrm{r}$ & 2 \\
\hline 329 & $\begin{array}{l}\text { So auh in andreru steid dhurah dhen selbun heilegun } \\
\text { forasagun uuard dhera dhrinissa bauhnunc sus } \\
\text { araughit: }\end{array}$ & $\mathrm{n}$ & 0 & $\mathrm{n}$ & 0 & 0 & 0 & s & $\mathrm{t}$ & 2 \\
\hline 336 & dhuo ir quhad: "Druhtines gheist ist ubar mir." & $\mathrm{n}$ & 0 & $\mathrm{n}$ & 0 & 0 & 0 & $s$ & $\mathrm{r}$ & 2 \\
\hline 338 & $\begin{array}{l}\text { Endi auh ir selbo Isaias in andreru stedi alle dhea } \\
\text { dhrinissa in fingro zalu bifenc, }\end{array}$ & $\mathrm{n}$ & 0 & $\mathrm{n}$ & 0 & 0 & 0 & 1 & r & 2 \\
\hline 346 & $\begin{array}{l}\text {.endi einnissa dhera almahtigun spuodi, } \\
\text { (dhurahchundida) }\end{array}$ & $\mathrm{e}$ & 1 & $\mathrm{n}$ & 0 & 0 & 0 & 0 & $\mathrm{r}$ & 3 \\
\hline 349 & $\begin{array}{l}\text { Dher selbo forasago auh in andrern stedi chundida, } \\
\text { dhazs ir dhera dhrinissa chiruni bichnadi, }\end{array}$ & $\mathrm{n}$ & 0 & $\mathrm{n}$ & 0 & 0 & 0 & 0 & $\mathrm{r}$ & 2 \\
\hline 363 & $\begin{array}{l}\text { endi dhoh eina guotliihhin dhera dhrinissa Syrafin } \\
\text { mit dhemu dhrifaldin quhide meinidon.... }\end{array}$ & $\mathrm{n}$ & 0 & $\mathrm{e}$ & 1 & 0 & 0 & 0 & $r$ & 3 \\
\hline 365 & $\begin{array}{l}\text {...Inu huuazs andres zeihnit dhar dhea dhri sanctus } \\
\text { chiquhedan, nibu dhera selbun almahtigun } \\
\text { dhrinissa guotliihhin ist araughit? ... }\end{array}$ & $\mathrm{e}$ & 2 & $\mathrm{n}$ & 0 & 0 & 0 & 0 & $\mathrm{t}$ & 4 \\
\hline
\end{tabular}


Tabelle 2: Meister Eckhart/synthetischer Genitiv/

\begin{tabular}{|c|c|c|c|c|c|c|c|c|c|c|}
\hline $\mathrm{S}$. & Text & $\mathrm{A}$ & $\mathrm{B}$ & $\mathrm{C}$ & $\mathrm{D}$ & E & $\mathrm{F}$ & $\mathrm{G}$ & $\mathrm{H}$ & $\mathrm{I}$ \\
\hline $\begin{array}{l}232 / \\
6 \\
\end{array}$ & $\begin{array}{l}\text { "gesegnet sî got und der vater unsers herren Jêsû } \\
\text { Kristî.,... }\end{array}$ & $\mathrm{e}$ & 3 & $\mathrm{e}$ & 0 & 0 & 1 & $\mathrm{~s}$ & $\mathrm{r}$ & 6 \\
\hline 6 & ...ein vater der barmherzicheit und & $\mathbf{n}$ & 0 & $\mathrm{n}$ & 0 & 0 & 0 & $s$ & $\mathrm{r}$ & 2 \\
\hline 6 & ...got alles trôstes, & e & 1 & $\mathrm{n}$ & 0 & 0 & 0 & $\mathrm{~s}$ & r & 3 \\
\hline 10 & Einez ist an dem schaden ûzerlîches guotes. & $\mathrm{e}$ & 1 & $\mathrm{n}$ & 0 & 0 & 1 & I & $\mathrm{r}$ & 3 \\
\hline 12 & $\begin{array}{l}\text { Daz dritte is an im selben an smâcheit, an } \\
\text { ungemache und an smerzen des libes und... }\end{array}$ & $\mathrm{n}$ & 0 & $\mathrm{e}$ & 0 & 0 & 0 & I & $\mathrm{r}$ & 4 \\
\hline 13 & .... an leide des herzen. & $\mathbf{n}$ & 0 & $\mathrm{n}$ & 0 & 0 & 0 & 1 & $\mathrm{r}$ & 2 \\
\hline 22 & $\begin{array}{l}\text { Her nâch vindet man in dem dritten teile dis } \\
\text { buoches bilde an werken }\end{array}$ & $\mathrm{e}$ & 1 & $\mathrm{e}$ & 1 & 0 & 0 & I & $\mathrm{r}$ & 4 \\
\hline $\begin{array}{l}234 / \\
5\end{array}$ & $\begin{array}{l}\text { ist ungemachet und ungeschaffen und doch geborn } \\
\text { kint und sun der güete }\end{array}$ & $\mathrm{n}$ & 0 & $\mathrm{e}$ & 0 & 0 & 0 & $\mathrm{~s}$ & r & 3 \\
\hline 9 & $\begin{array}{l}\text { und der guote nimet allez sîn wesen, wizzen, minnen } \\
\text { und würken von dem herzen und innigesten der } \\
\text { güete }\end{array}$ & $\mathrm{n}$ & 0 & $\mathrm{n}$ & 0 & 0 & 0 & I & $\mathrm{r}$ & 2 \\
\hline $\begin{array}{l}236 / \\
4 \\
\end{array}$ & $\begin{array}{l}\text { Allez, daz ich nû hân gesprochen (...) von gotes } \\
\text { sune, }\end{array}$ & $\mathrm{n}$ & 0 & $\mathrm{n}$ & 0 & 0 & 0 & I & $r$ & 2 \\
\hline 10 & $\begin{array}{l}\text { daz "allen den ist gegeben maht und mugent, gotes } \\
\text { suine zu werdenne, ... }\end{array}$ & $\mathrm{n}$ & 0 & $\mathrm{n}$ & 0 & 0 & 0 & I & I & 2 \\
\hline 14 & $\begin{array}{l}\text { daz an dem menschen niht underænic ist des } \\
\text { menschen willen. }\end{array}$ & $\mathrm{n}$ & 0 & $\mathrm{n}$ & 0 & 0 & 0 & $\mathrm{~s}$ & $\mathrm{r}$ & 2 \\
\hline 14 & Bî des vleisches willen meinet er allez, .. & $\mathrm{n}$ & 0 & $\mathrm{n}$ & 0 & 0 & 0 & I & $\mathbf{t}$ & 2 \\
\hline 17 & und (er) neiget nâch des vleisches begerunge & $\mathbf{n}$ & 0 & $\mathrm{n}$ & 0 & 0 & 0 & I & r & 2 \\
\hline 20 & Bei dem willen des mannes meinet sant Johannes... & $\mathrm{n}$ & 0 & $\mathrm{n}$ & 0 & 0 & 0 & 1 & $\mathrm{t}$ & 2 \\
\hline 20 & ...die hhsten krefte sêle, der nature. & $\mathrm{e}$ & 0 & $\mathrm{e}$ & 1 & 1 & 0 & o & $\mathrm{r}$ & 4 \\
\hline 22 & und (sie) stânt in der sêle lûterkeit, & $\mathrm{n}$ & 0 & $\mathrm{n}$ & 0 & 0 & 0 & 1 & r & 2 \\
\hline 26 & in den der mensche gotes geslehte ist ... & $\mathrm{n}$ & 0 & $\mathbf{n}$ & 0 & 0 & 0 & 1 & $\mathbf{r}$ & 2 \\
\hline 31 & ...und gotes sippe. & $\mathrm{n}$ & 0 & $\mathrm{n}$ & 0 & 0 & 0 & I & r & 2 \\
\hline 31 & wan alsô sint sie ouch gotes süne und ... & $\mathrm{n}$ & 0 & $\mathrm{n}$ & 0 & 0 & 0 & 1 & $r$ & 2 \\
\hline 31 & ...gotes eingeborn sun. & $\mathrm{n}$ & 0 & $\mathrm{e}$ & 1 & 0 & 0 & 1 & $\mathbf{r}$ & 3 \\
\hline 33 & Ein sôgetân mensche, gote & $\mathrm{n}$ & 0 & n & 0 & 0 & 0 & $\mathrm{~s}$ & $\mathrm{t}$ & 2 \\
\hline \begin{tabular}{|l|}
$236 /$ \\
33 \\
\end{tabular} & ...guot der güete sun, ... & $\mathbf{n}$ & 0 & $\mathrm{e}$ & 1 & 0 & 0 & $s$ & $\mathrm{t}$ & 3 \\
\hline $238 / 1$ & $\begin{array}{l}\text {...gereht sun der gerehtichkeit, alsô verre als er } \\
\text { aleine ir sun ist, }\end{array}$ & $\mathrm{n}$ & 0 & $\mathrm{e}$ & 1 & 0 & 0 & s & $\mathrm{t}$ & 3 \\
\hline 4 & $\begin{array}{l}\text { und (er) tritet in alle die eigenschaft der } \\
\text { gerehtichkeit und der wârheit. }\end{array}$ & $\mathrm{e}$ & 0 & $\mathrm{e}$ & 1 & 0 & 0 & I & $\mathrm{r}$ & 4 \\
\hline 7 & $\begin{array}{l}\text { diu in dem heiligen êwangeliô geschriben ist und } \\
\text { sicherlîche bekant in dem natiurlichen liehte der } \\
\text { vernünftigen sêle, }\end{array}$ & $\mathrm{e}$ & 1 & $\mathrm{e}$ & 1 & 0 & 0 & 1 & $\mathrm{r}$ & 4 \\
\hline $\begin{array}{l}240 / \\
20 \\
\end{array}$ & und (er blîbet) in vride sînes herzen unbeweget, & e & 1 & $\mathrm{n}$ & 0 & 0 & 1 & I & $\mathrm{r}$ & 3 \\
\hline 26 & Ist ez danne reht gotes, & $\mathrm{n}$ & 0 & $\mathrm{n}$ & 0 & 0 & 0 & I & $\mathrm{r}$ & 2 \\
\hline
\end{tabular}




\begin{tabular}{|c|c|c|c|c|c|c|c|c|c|c|}
\hline $\begin{array}{l}242 / \\
10 \\
\end{array}$ & $\begin{array}{l}\text { Mîn herze und mîn minne gibet die güete der } \\
\text { crêatûre, }\end{array}$ & $\mathrm{n}$ & 0 & $\mathrm{n}$ & 0 & 0 & 0 & 0 & $r$ & 2 \\
\hline 18 & Diz sî nû genuoc von dem êrsten teile dis buoches. & $\mathrm{e}$ & 1 & $\mathrm{e}$ & 1 & 0 & 0 & 1 & $\mathrm{r}$ & 4 \\
\hline 26 & $\begin{array}{l}\text { Dar umbe sprichet sant Paulus, daz gotes triuwe } \\
\text { und güete enlîdet des niht }\end{array}$ & $\mathrm{n}$ & 0 & $\mathrm{e}$ & 0 & 0 & 0 & $\mathrm{~s}$ & $\mathrm{r}$ & 3 \\
\hline $\begin{array}{l}244 / \\
15\end{array}$ & "in den tagen des leides vergiz niht ... . & $\mathrm{n}$ & 0 & $\mathrm{n}$ & 0 & 0 & 0 & I & $\mathrm{t}$ & 2 \\
\hline 15 & ...der tage der güete". & $\mathrm{n}$ & 0 & $\mathrm{n}$ & 0 & 0 & 0 & $\mathrm{o}$ & $\mathrm{r}$ & 2 \\
\hline 24 & Ist er siech und in grôzem smerzen sînes lîbes, & $\mathrm{e}$ & 1 & $\mathrm{e}$ & 1 & 0 & 1 & 1 & $\mathrm{r}$ & 4 \\
\hline 25 & (er hat nôtdurft) an râte der arzete.. & $\mathrm{n}$ & 0 & $\mathrm{n}$ & 0 & 0 & 0 & 1 & $\mathrm{r}$ & 2 \\
\hline 26 & ...und an dienste sînes gesindes & $\mathrm{e}$ & 1 & $\mathrm{n}$ & 0 & 0 & 1 & 1 & $r$ & 3 \\
\hline 27 & ...an klage und an bîwessene sîner vriunde: & $\mathrm{e}$ & 1 & $\mathrm{e}$ & 0 & 0 & 1 & 1 & $\mathbf{r}$ & 4 \\
\hline $\begin{array}{l}246 / \\
11\end{array}$ & $\begin{array}{l}\text { daz man mit dir, der wârheit, valscheit und trüge } \\
\text { der crêatûren (...) besitze. }\end{array}$ & $\mathrm{n}$ & 0 & e & 0 & 0 & 0 & I & $\mathrm{r}$ & 4 \\
\hline 14 & $\begin{array}{l}\text { Und anderswâ sprichet er: "wie möhte dem } \\
\text { genuiegen an gotes gâben }\end{array}$ & $\mathbf{n}$ & 0 & $\mathrm{n}$ & 0 & 0 & 0 & 1 & $\mathbf{r}$ & 2 \\
\hline 27 & der munt des menschen sprichet von dem & $\mathrm{n}$ & 0 & $\mathrm{n}$ & 0 & 0 & 0 & $\mathrm{~s}$ & $\mathrm{t}$ & 2 \\
\hline 29 & daz "der munt sprichet von der vülle des herzen", & $\mathrm{n}$ & 0 & $\mathrm{n}$ & 0 & 0 & 0 & 1 & $\mathrm{r}$ & 2 \\
\hline 32 & sunder din crêatûre ist in des menschen herzen & $\mathrm{n}$ & 0 & $\mathrm{n}$ & 0 & 0 & 0 & 1 & $\mathrm{r}$ & 2 \\
\hline $\begin{array}{l}248 / \\
28\end{array}$ & und wir biten alle tage, daz gotes wille gewerde. & $\mathrm{n}$ & 0 & $\mathrm{n}$ & 0 & 0 & 0 & $\mathbf{s}$ & $\mathrm{r}$ & 2 \\
\hline 28 & Und do & $\mathrm{n}$ & 0 & n & 0 & 0 & 0 & $s$ & $\mathrm{t}$ & 2 \\
\hline \begin{tabular}{|l|l}
$250 /$ \\
3 \\
\end{tabular} & $\begin{array}{l}\text { Ez sprichet ein heidenischer Meister: herzoge und } \\
\text { oberster vater und herre des hôhen himels, }\end{array}$ & $\mathrm{e}$ & 1 & $\mathrm{n}$ & 0 & 0 & 0 & $s$ & $\mathrm{r}$ & 3 \\
\hline 12 & dâ gotes êre breiter und grzlîcher ane læge. & $\mathrm{n}$ & 0 & $\mathrm{n}$ & 0 & 0 & 0 & $s$ & $\mathrm{t}$ & 2 \\
\hline 13 & daz er gotes wille ist, & $\mathrm{n}$ & 0 & $\mathrm{n}$ & 0 & $\underline{0}$ & 0 & 1 & $r$ & 2 \\
\hline 14 & sô sol des guoten menschen wille alsô gar mit ... & $\mathrm{e}$ & 1 & $\mathrm{n}$ & 0 & 0 & 0 & $s$ & $\mathrm{r}$ & 3 \\
\hline 14 & ...gotes willen ein und geeiniget sîn, & $\mathrm{n}$ & 0 & $\mathrm{n}$ & 0 & 0 & 0 & 1 & $r$ & 2 \\
\hline 18 & $\begin{array}{l}\text { daz er von gote gesundert wære durch got und durch } \\
\text { gotes willen }\end{array}$ & $\mathrm{n}$ & 0 & e & 0 & 0 & 0 & I & $\mathrm{r}$ & 2 \\
\hline
\end{tabular}

\section{Tabelle 3: Martin Luther /synthetischer Genitiv/}

\begin{tabular}{|l|l|c|c|c|c|c|c|c|c|c|}
\hline S. & Text & A & B & C & D & E & F & G & H & I \\
\hline $\begin{array}{l}112 / \\
2\end{array}$ & Eur löblichen Stadt Prediger hat mir hoch gepreiset & e & 2 & n & 0 & 0 & 1 & s & t & 4 \\
\hline $\begin{array}{l}113 / \\
17\end{array}$ & $\begin{array}{l}\text { Diese zwo widerständige Rede der Freiheit und } \\
\text { Dienstbarkeit zu vernehmen, sollen wir gedenken }\end{array}$ & e & 0 & e & 3 & 0 & 0 & o & $/$ & 5 \\
\hline $\begin{array}{l}114 / \\
22\end{array}$ & $\begin{array}{l}\text { Zum funften hat die Seele kein ander Ding, (...) denn } \\
\text { das heilig Evangelii, das Wort Gottes }\end{array}$ & n & 0 & $\mathrm{n}$ & 0 & 0 & 0 & o & r & 2 \\
\hline 28 & die do gehen von dem Mund Gottes & $\mathrm{n}$ & 0 & $\mathrm{n}$ & 0 & 0 & 0 & $/$ & $\mathrm{r}$ & 2 \\
\hline 29 & $\begin{array}{l}\text { So mussen wir nu gewiß sein, daß die Seele kann } \\
\text { alles Dings, ohn das Wort Gottes ... }\end{array}$ & $\mathrm{n}$ & 0 & $\mathrm{n}$ & 0 & 0 & 0 & $/$ & $\mathrm{r}$ & 2 \\
\hline 30 & $\begin{array}{l}\text {...und ohn das Wort Gottes ist ihr mit keinem Ding } \\
\text { geholfen }\end{array}$ & $\mathrm{n}$ & 0 & $\mathrm{n}$ & 0 & 0 & 0 & $/$ & $\mathrm{t}$ & 2 \\
\hline
\end{tabular}




\begin{tabular}{|c|c|c|c|c|c|c|c|c|c|c|}
\hline $\begin{array}{ll}115 / \\
2\end{array}$ & $\begin{array}{l}\text { und in der Schrift die allerhochste Plag und Gottes } \\
\text { Zorn gehalten wird }\end{array}$ & $\mathrm{n}$ & 0 & n & 0 & 0 & 0 & o & $\mathrm{r}$ & 2 \\
\hline 7 & $\begin{array}{l}\text { Und Christus um keins andern Amts willen, denn zu } \\
\text { predigen das Wort Gottes, kummen ist. }\end{array}$ & $\mathrm{n}$ & 0 & $\mathrm{n}$ & 0 & 0 & 0 & o & 1 & 2 \\
\hline 21 & $\begin{array}{l}\text { so mußt du an dir selber verzweifeln und bekennen, } \\
\text { daß wahr sei der Spruch Hoseae }\end{array}$ & $\mathrm{n}$ & 0 & $\mathrm{n}$ & 0 & 0 & 0 & $\mathrm{~s}$ & $\mathrm{r}$ & 2 \\
\hline 33 & Christus ist das Ende und Fülle aller Gebot denen & $\mathrm{e}$ & 1 & $\mathrm{e}$ & 0 & 0 & 0 & 1 & $\mathrm{r}$ & 4 \\
\hline 36 & 1 & $\mathrm{e}$ & 1 & $\mathrm{e}$ & 1 & 0 & 0 & 1 & $r$ & 5 \\
\hline $\begin{array}{ll}116 / \\
16\end{array}$ & in kurzlich aller Gehot Frfullung & $\mathrm{e}$ & 1 & $\mathrm{n}$ & 0 & 0 & 0 & $\mathrm{~s}$ & $r$ & 3 \\
\hline 31 & $\begin{array}{l}\text { daß die ganze Heilige Schrift wird in zweierlei Wort } \\
\text { geteilt, wilche sind: Gebot oder Gesetz Gottes und } \\
\text { Verheischen oder Zusagunge }\end{array}$ & $\mathrm{n}$ & 0 & $\mathrm{e}$ & 0 & 0 & 0 & $\mathrm{~s}$ & $r$ & 3 \\
\hline $\begin{array}{l}117 / \\
22\end{array}$ & & $\mathrm{n}$ & 0 & $\mathrm{e}$ & 1 & 0 & 0 & 1 & r & 3 \\
\hline 28 & & $\mathrm{n}$ & 0 & $n$ & 0 & 0 & 0 & 0 & $r$ & 2 \\
\hline $\begin{array}{l}118 / \\
1\end{array}$ & $\begin{array}{l}\text { wer ihn' mit einem rechten Glauben anhangt, des } \\
\text { Seele wird mit ihm vereinigt so ganz und gar, daß } \\
\text { alle Tugend des Worts auch eigen werden der } \\
\text { Seelen ... }\end{array}$ & $\mathrm{n}$ & 0 & $\mathrm{e}$ & 1 & 0 & 0 & s & $\mathrm{r}$ & 3 \\
\hline 4 & $\begin{array}{l}\text {...und also durch den Glauben die Seele von dem } \\
\text { Gotteswort heilig, gerecht, wahrhaftig, friedsam, frei } \\
\text { und aller Güte voll, ein wahrhaftig Kind Gottes } \\
\text { wird }\end{array}$ & $\mathrm{n}$ & 0 & $\mathrm{e}$ & 1 & 0 & 0 & 1 & $r$ & 3 \\
\hline 29 & & $\mathrm{n}$ & 0 & $\mathrm{n}$ & 0 & 0 & 0 & 0 & $\mathrm{r}$ & 2 \\
\hline $\begin{array}{ll}119 / \\
3\end{array}$ & $\begin{array}{l}\text { ihn verleugne } \\
\text { Abgott ihres } \\
\text { aufricht, } \\
\end{array}$ & $\mathrm{e}$ & 2 & $n$ & 0 & 0 & 1 & 0 & $r$ & 4 \\
\hline 27 & & $\mathrm{e}$ & 1 & $\mathrm{n}$ & 0 & 0 & 0 & 0 & $\mathrm{r}$ & 3 \\
\hline 34 & & $\mathrm{e}$ & 2 & $\mathrm{e}$ & 1 & 0 & 1 & 1 & $\mathrm{r}$ & 5 \\
\hline $\begin{array}{l}120 / \\
21\end{array}$ & $\begin{array}{l}\text { Das tu } \\
\text { Glaub }\end{array}$ & $\mathrm{n}$ & 0 & $\mathrm{n}$ & 0 & 0 & 0 & $s$ & $\mathrm{r}$ & 2 \\
\hline 22 & die Gerechtigkeit des & $\mathrm{n}$ & 0 & $\mathrm{n}$ & 0 & 0 & 0 & 1 & $r$ & 2 \\
\hline 23 & & $\mathrm{e}$ & 1 & $\mathrm{n}$ & 0 & 0 & 0 & 1 & $r$ & 3 \\
\hline 30 & Das ist & $\mathrm{n}$ & 0 & $\mathrm{n}$ & 0 & 0 & 0 & 1 & $r$ & 2 \\
\hline 31 & $\begin{array}{l}\text {...der ist das Haupt und ganzes Wesen der } \\
\text { Frummkeit }\end{array}$ & $\mathrm{n}$ & 0 & $\mathrm{e}$ & 1 & 0 & 0 & I & $\mathrm{r}$ & 4 \\
\hline $\begin{array}{ll}121 / \\
22 \\
\end{array}$ & $\begin{array}{l}\text { also daß er fur Gottes Augen ohn Unterlaß fur die } \\
\text { Seinen steht }\end{array}$ & $\mathrm{n}$ & 0 & $\mathrm{n}$ & 0 & 0 & 0 & 1 & $r$ & 2 \\
\hline 26 & & $\mathrm{n}$ & 0 & $\mathrm{e}$ & 3 & 0 & 0 & 1 & $r$ & 5 \\
\hline $\begin{array}{l}122 / \\
22\end{array}$ & $\begin{array}{l}\text { Siehe, wie ist das ein köstlich FI } \\
\text { der Christen. }\end{array}$ & $\mathrm{n}$ & 0 & $\mathrm{e}$ & 1 & 0 & 0 & 1 & $r$ & 4 \\
\hline
\end{tabular}




\begin{tabular}{|c|c|c|c|c|c|c|c|c|c|c|}
\hline 26 & Denn fur Gottes Augen zu stehen und bitten, & $\mathrm{n}$ & 0 & $\mathrm{n}$ & 0 & 0 & 0 & 1 & 1 & 2 \\
\hline 31 & $\begin{array}{l}\text { Wer aber nit glaubt an Christum, ist ein Knecht } \\
\text { aller Ding, ... }\end{array}$ & $\mathrm{e}$ & 1 & $\mathrm{n}$ & 0 & 0 & 0 & 1 & $\mathrm{r}$ & 3 \\
\hline 32 & & $\mathrm{n}$ & 0 & $\mathrm{n}$ & 0 & 0 & 0 & 1 & $r$ & 2 \\
\hline 33 & & $\mathrm{n}$ & 0 & $\mathrm{e}$ & 0 & 0 & $\overline{0}$ & o & $\mathrm{r}$ & 3 \\
\hline $\begin{array}{l}123 / \\
28\end{array}$ & $\begin{array}{l}\text { Wir wollen nichts mehr von den Leuten gehalten } \\
\text { sein denn Christus Diener und Schaffner des } \\
\text { Evangelii. }\end{array}$ & $\mathrm{n}$ & 0 & $\mathrm{e}$ & 0 & 0 & 0 & 1 & $\mathrm{r}$ & 3 \\
\hline 33 & $\begin{array}{l}\text { die Laien etwas anders denn Christenleut, } \\
\text { renummen ist der ganz Verstand } \\
\text { er Gnad, Freiheit, Glaubens und alles }\end{array}$ & $\mathrm{e}$ & 1 & $\mathrm{e}$ & 1 & 0 & 0 & $\mathrm{~s}$ & $\mathrm{r}$ & 6 \\
\hline \begin{tabular}{l|l|}
$124 /$ \\
1
\end{tabular} & $\begin{array}{l}\text { el Menschen Gesetz und } \\
\text { rden der } \\
\text { Erden. }\end{array}$ & $\mathrm{e}$ & 1 & $\mathrm{e}$ & 1 & 1 & 0 & 1 & $r$ & 4 \\
\hline 4 & & $\mathrm{n}$ & 0 & $\mathrm{e}$ & 0 & 0 & 0 & 0 & $r$ & 3 \\
\hline 7 & $\begin{array}{l}\text { so man sein gar schweigt und da } \\
\text { oder ander Menschen Gesetz } u\end{array}$ & $\mathrm{e}$ & 1 & $\mathrm{e}$ & 0 & 0 & 0 & 0 & $\mathrm{r}$ & 4 \\
\hline 18 & $\begin{array}{l}\text { was wir } \\
\text { erhöret s }\end{array}$ & $\mathrm{n}$ & 0 & $\mathrm{n}$ & 0 & 0 & 0 & 1 & $r$ & 2 \\
\hline 25 & $\begin{array}{l}\text { Frummkeit sei sein und sein Sund sein } \\
\text { in }\end{array}$ & $\mathrm{n}$ & 0 & $\mathrm{n}$ & 0 & 0 & 0 & $\mathrm{~s}$ & $t$ & 2 \\
\hline 27 & $\begin{array}{l}\text { so mu } \\
\text { Frum }\end{array}$ & $\mathrm{n}$ & 0 & $\mathrm{n}$ & 0 & 0 & 0 & 1 & $\mathrm{r}$ & 2 \\
\hline $\begin{array}{ll}125 / \\
15\end{array}$ & $\begin{array}{l}\text { Daher } \\
\text { sein " }\end{array}$ & $\mathrm{n}$ & 0 & $\mathrm{e}$ & 1 & 0 & 0 & 1 & $r$ & 3 \\
\hline $\begin{array}{ll}126 / \\
4\end{array}$ & $\begin{array}{l}\text { Ich hab ein Lust in Gottes Willen nach mein } \\
\text { innern Menschen }\end{array}$ & $\mathrm{n}$ & 0 & $\mathrm{n}$ & 0 & 0 & 0 & 1 & 1 & 2 \\
\hline $\begin{array}{ll}127 / \\
2\end{array}$ & Torheit und Unverstand & $\mathrm{e}$ & 1 & $\mathrm{e}$ & 1 & 0 & 0 & 1 & $\mathrm{I}$ & 6 \\
\hline 5 & $\begin{array}{l}\text { soll man die Werk eines Christenmenschen } \\
\text { anders achten) }\end{array}$ & $\mathrm{n}$ & 0 & $\mathrm{n}$ & 0 & 0 & 0 & 0 & $\mathrm{r}$ & 2 \\
\hline 19 & $\begin{array}{l}\text { Also auch eines glaubigen Menschen Werk (darf } \\
\text { keiner Werk sondern) }\end{array}$ & $\mathrm{e}$ & 1 & $\mathrm{n}$ & 0 & 0 & 0 & 0 & $\mathrm{t}$ & 3 \\
\hline 26 & $\begin{array}{l}\text { wenn der Kirchen weihet, fermelt oder sonst seines } \\
\text { Amts Werk ubet, }\end{array}$ & $\mathrm{e}$ & 1 & $\mathrm{n}$ & 0 & 0 & 1 & 0 & $r$ & 3 \\
\hline 32 & (wilch nit denn des Glaubens Mehrung tut) & $\mathrm{n}$ & 0 & $\mathrm{n}$ & 0 & 0 & 0 & 0 & I & 2 \\
\hline $\begin{array}{l}128 / \\
24\end{array}$ & Also sein die Werk des Menschen auch: & $\mathrm{n}$ & 0 & $\mathrm{n}$ & 0 & 0 & 0 & $\mathrm{~s}$ & $t$ & 2 \\
\hline
\end{tabular}


Tabelle 4: Abraham a Sancta Clara/synthetischer Genitiv/

\begin{tabular}{|c|c|c|c|c|c|c|c|c|c|c|}
\hline Zeile & Text & $\mathrm{A}$ & $\mathrm{B}$ & $\mathrm{C}$ & D & $\mathrm{E}$ & $\mathrm{F}$ & $\mathrm{G}$ & $\mathrm{H}$ & I \\
\hline $9 / 2$ & $\begin{array}{l}\text { ALergnädigiste Mayestätten/ nunmehr gewint heut } \\
\text { zur Trost der gantzen Christenheit }\end{array}$ & $\mathrm{e}$ & 1 & $\mathrm{n}$ & 0 & 0 & 0 & I & $r$ & 3 \\
\hline 7 & $\begin{array}{l}\text { indeme Euere Mayest. als im Firmament deß Röm. } \\
\text { Reichs schönst-strahlende Sonn und Mond ... }\end{array}$ & $\mathrm{e}$ & 1 & $n$ & 0 & 0 & 0 & 1 & $\mathbf{r}$ & 3 \\
\hline 9 & ...den glorreichesten Ertz-Vatter Christi/ & $\mathbf{n}$ & 0 & e & 1 & 1 & 0 & 0 & $r$ & 3 \\
\hline 9 & $\begin{array}{l}\text {..Gespons Mariae/Joseph/ mit höchtsgepriesenen } \\
\text { Eiffer heut verehren }\end{array}$ & $\mathbf{n}$ & 0 & $\mathrm{n}$ & 0 & 0 & 0 & 0 & $\mathrm{r}$ & 2 \\
\hline 15 & $\begin{array}{l}\text { weilen nun dieser trostreiche Pfleg-Vatter mehresten } \\
\text { Theil vorgebildet wird mit einer schneeweissen } \\
\text { Lilien in der Hand zum sichbarlichen Kehnzeichen } \\
\text { seiner unversehrten Jungfräulichen Reinigkeit/ } \\
\text { (wollen praesentieren) }\end{array}$ & $\mathrm{n}$ & 3 & $\mathrm{e}$ & 1 & 0 & 1 & 1 & I & 6 \\
\hline 22 & $\begin{array}{l}\text { jedoch ist gäntzlich zu hoffen/ daß noch einmal } \\
\text { durch den Willen Gottes werde begegnen/ }\end{array}$ & $\mathrm{n}$ & 0 & $\mathrm{n}$ & 0 & 0 & 0 & I & $\mathrm{I}$ & 2 \\
\hline $\begin{array}{l}10 / \\
14 \\
\end{array}$ & $\begin{array}{l}\text { zumalen sie auß dem Hof-Garten ist deß } \\
\text { Allerhöchsten GOTT/ }\end{array}$ & $\mathrm{e}$ & 1 & $\mathrm{n}$ & 0 & 1 & 0 & I & $\mathbf{r}$ & 3 \\
\hline $11 / 8$ & $\begin{array}{l}\text { Aus allen Blumen der Welt har Er ihme erwehlt die } \\
\text { Lilien/ }\end{array}$ & $\mathbf{n}$ & 0 & $\mathrm{e}$ & 1 & 0 & 0 & 1 & $t$ & 3 \\
\hline 12 & $\begin{array}{l}\text { künd ich euch samentlich an ihr Gottgesegnete Erb- } \\
\text { Kronen und Erb-Provintzen deß } \\
\text { Allerdurchleuchtigsten Ertz-Haus Oesterreichs: }\end{array}$ & $\mathrm{e}$ & 2 & $\mathrm{e}$ & 2 & 1 & 1 & 1 & $r$ & 7 \\
\hline 17 & $\begin{array}{l}\text { welchen der auserwöhlte Gärtmer Namens JESU } \\
\text { von Nazareth mit seinem Creutz umbgraben/ }\end{array}$ & e & 1 & e & 1 & 0 & 0 & $s$ & $\mathrm{r}$ & 4 \\
\hline 23 & $\begin{array}{l}\text { In diesem Garten der Catholischen Kirchen ist } \\
\text { gewachsen (Schlüssel-Blum) }\end{array}$ & $\mathrm{e}$ & 1 & $\mathrm{e}$ & 1 & 0 & 0 & 1 & $\mathrm{t}$ & 4 \\
\hline 28 & Wer nun begehrt/ deß Himmels Werth/ & $\mathrm{n}$ & 0 & $\mathrm{n}$ & 0 & 0 & 0 & 0 & $\mathrm{r}$ & 2 \\
\hline $12 / 1$ & $\begin{array}{l}\text { In diesem Garten der Römischen Kirchen ist } \\
\text { gewachsen (Blum) }\end{array}$ & $\mathrm{e}$ & 1 & $\mathrm{e}$ & 1 & 0 & 0 & / & $\mathrm{t}$ & 4 \\
\hline 9 & $\begin{array}{l}\text { In diesem Garten der Apostolischen Kirchen ist } \\
\text { gewachsen/ (Blümlein) }\end{array}$ & e & 1 & $\mathrm{e}$ & 1 & 0 & 0 & 1 & $\mathrm{t}$ & 4 \\
\hline 17 & $\begin{array}{l}\text { In diesem Garten der allein-seligmachenden } \\
\text { Kirchen ist gewachsen/ (Majoran) }\end{array}$ & $\mathrm{e}$ & 1 & $\mathrm{e}$ & 1 & 0 & 0 & I & $t$ & 4 \\
\hline 26 & $\begin{array}{l}\text { In diesem Garten der allzeit-florirenden Kirchen } \\
\text { ist gewachsen/ (Ritter-Sporn) }\end{array}$ & $\mathrm{e}$ & 2 & e & 1 & 0 & 0 & 1 & $\mathrm{t}$ & 4 \\
\hline $13 / 1$ & $\begin{array}{l}\text { In diesem Garten der allzeit regierenden Kirchen } \\
\text { ist gewachsen/(Lavandel) }\end{array}$ & e & 2 & $\mathrm{e}$ & 1 & 0 & 0 & 1 & $t$ & 5 \\
\hline 4 & $\begin{array}{l}\text { als die ihr gantze Krafft und Safft durch die } \\
\text { Benetzung der bußfertigen Zäher erlangt/ } \\
\end{array}$ & $\mathrm{e}$ & 1 & $\mathrm{n}$ & 0 & 0 & 0 & 1 & $r$ & 3 \\
\hline 10 & $\begin{array}{l}\text { In diesem Garten der Catholischen Kirchen ist } \\
\text { gewachsen/ ... (Rosa Mystica) }\end{array}$ & $\mathrm{e}$ & 1 & e & 1 & 0 & 0 & 1 & $\mathrm{t}$ & 4 \\
\hline 16 & $\begin{array}{l}\text { aus dero die Himmlische Bühn Christus das Hönig } \\
\text { seiner Menschheit genommen/ }\end{array}$ & $\mathrm{n}$ & 1 & $\mathrm{n}$ & 0 & 0 & 1 & 0 & $\mathbf{r}$ & 3 \\
\hline 21 & $\begin{array}{l}\text { In diesem Garten der Catholischen Kirchen seyn } \\
\text { unzahlbare (...) gewachsen (Blumen) }\end{array}$ & e & 1 & e & 1 & 0 & 0 & 1 & $t$ & 4 \\
\hline 25 & $\begin{array}{l}\text { Dort wie viel seynd schönste Veigeln der } \mathbf{H} \text {. } \\
\text { Patriarchen/ ... }\end{array}$ & $\mathrm{e}$ & 1 & $\mathrm{e}$ & 1 & 1 & 0 & $s$ & $r$ & 4 \\
\hline
\end{tabular}




\begin{tabular}{|c|c|c|c|c|c|c|c|c|c|c|}
\hline 26 & $\begin{array}{l}\text {...wie viel schönste Sonnenwendt der H. } \\
\text { Propheten/ ... }\end{array}$ & $\mathrm{e}$ & 1 & $\mathrm{e}$ & 1 & 1 & 0 & $\mathrm{~s}$ & $\mathrm{r}$ & 4 \\
\hline 27 & ...wie viel schönste Tullibanen der H. Aposteln/ & $\mathrm{e}$ & 1 & $\mathrm{e}$ & 1 & 1 & 0 & $s$ & $\mathbf{r}$ & 4 \\
\hline 28 & $\begin{array}{l}\text {... wie viel schönste Wohlgemuth der H. Martyrer/ } \\
\text {... }\end{array}$ & $\mathrm{e}$ & 1 & e & 1 & 1 & 0 & $\mathrm{~s}$ & $\mathbf{r}$ & 4 \\
\hline 29 & $\begin{array}{l}\text {...wie viel schönste Tag und Nacht der } H \text {. } \\
\text { Beleuchtiger/ ... }\end{array}$ & $\mathrm{e}$ & 1 & $\mathrm{e}$ & 1 & 1 & 0 & $s$ & $\mathrm{r}$ & 5 \\
\hline 30 & $\begin{array}{l}\text {... wie viel schönste weisse Narcissen der } \mathbf{H} \text {. } \\
\text { Jungfrauen/ ... }\end{array}$ & $\mathrm{e}$ & 1 & $\mathrm{e}$ & 2 & 1 & 0 & $\mathrm{~s}$ & $\mathrm{r}$ & 5 \\
\hline 31 & $\begin{array}{l}\text {..wie viel schönste Vergiß mein nit der } \mathbf{H} \text {. } \\
\text { Wittiben/ ... }\end{array}$ & $\mathrm{e}$ & 1 & $\mathrm{e}$ & 1 & 1 & 0 & $\mathrm{~s}$ & $\mathrm{r}$ & 4 \\
\hline 32 & ...wie viel schönste Ringl-Blumen der H. Eheleuth/ & $\mathrm{e}$ & 1 & $\mathrm{e}$ & 1 & 1 & 0 & $\mathrm{~s}$ & $\mathrm{r}$ & 4 \\
\hline 34 & $\begin{array}{l}\text { so allessambt (blïhende Blumen) durch den einigen } \\
\text { Anblick Göttelicher Sonnen-Strahlen auff ewig nit } \\
\text { verwelcken noch abfallen. }\end{array}$ & $\mathrm{e}$ & 1 & $\mathrm{e}$ & 1 & 0 & 0 & 1 & $\mathrm{r}$ & 4 \\
\hline $14 / 5$ & $\begin{array}{l}\text { aus allen diesen schönsten Blumen hat } \\
\text { LEOPOLDVS den zwölfften Tag deß Blumen- } \\
\text { Monats May (...) ... }\end{array}$ & $\mathrm{e}$ & 1 & $\mathrm{e}$ & 1 & 0 & 0 & o & $\mathrm{r}$ & 4 \\
\hline 7 & ...durch Eingebung Gottes $/ . .$. & $\mathrm{n}$ & 0 & $\mathrm{n}$ & 0 & 0 & 0 & 1 & $\mathrm{r}$ & 2 \\
\hline 7 & g deß Himmels/. & $\mathrm{n}$ & 0 & $\mathrm{n}$ & 0 & 0 & 0 & 1 & $\mathrm{r}$ & 2 \\
\hline 8 & ung aller Engel/.. & $\mathrm{e}$ & 1 & $\mathrm{n}$ & 0 & 0 & 0 & 1 & $\mathrm{r}$ & 3 \\
\hline 9 & ...mit Frolockung deß Volks/ (...) gezehlt/ & $\mathrm{n}$ & 0 & $\mathrm{n}$ & 0 & 0 & 0 & 1 & $r$ & 2 \\
\hline 19 & $\begin{array}{l}\text { LEOPOLDUS (...) das häuffige Hönig der Gnaden } \\
\text { zu saugen verhoffet/ }\end{array}$ & $\mathrm{n}$ & 0 & $\mathrm{e}$ & 1 & 0 & 0 & o & 1 & 3 \\
\hline 22 & $\begin{array}{l}\text { Eilet nach dem Geruch diser Himmlischen Lilien } \\
\text { Joseph, singet und klinget/ }\end{array}$ & $\mathrm{e}$ & 2 & $\mathrm{n}$ & 0 & 0 & 0 & 1 & $\mathrm{r}$ & 4 \\
\hline 26 & $\begin{array}{l}\text { SO wohl durch öfftern Augenschein wie nit weniger } \\
\text { durch des witzigen Plinii Aussag ist allen bekant/ }\end{array}$ & $\mathrm{e}$ & 1 & $\mathrm{n}$ & 0 & 0 & 0 & 1 & $\mathrm{r}$ & 3 \\
\hline $\begin{array}{l}15 / \\
10\end{array}$ & $\begin{array}{l}\text { Wer das letzte Capitul deß H. Evangelisten } \\
\text { Matthaei durchsuchet/ }\end{array}$ & $\mathrm{e}$ & 2 & $\mathrm{e}$ & 1 & 0 & 0 & 0 & $\mathrm{r}$ & 5 \\
\hline 15 & $\begin{array}{l}\text { wie der gebenedeyte HErr mit seinen geliebten drey } \\
\text { Jüngern auff den hohen Berg Thabro gestiegen/ und } \\
\text { ihnen alldorten einen kleinen Abriß seiner Glory } \\
\text { gezeigt/ }\end{array}$ & $\mathrm{e}$ & 1 & $\mathrm{e}$ & 1 & 0 & 1 & 0 & $\mathrm{r}$ & 4 \\
\hline 19 & $\begin{array}{l}\text { auch als Magdalena die zwey Engel nach der } \\
\text { Urstend Christi angetroffen bey dem Grab/ }\end{array}$ & $\mathrm{n}$ & 0 & $\mathrm{n}$ & 0 & 0 & 0 & 1 & $r$ & 2 \\
\hline 25 & $\begin{array}{l}\text { als hat er deßwegen den glorwürdigsten Joseph } \\
\text { diese schneeweisse Lilien der reinen } \\
\text { Jungfrauschafft und Jungfräulichen Reinigkeit } \\
\text { aus der ganzen Welt erwehlt ... } \\
\end{array}$ & $\mathrm{e}$ & 2 & $\mathrm{e}$ & 2 & 0 & 0 & 0 & $\mathrm{r}$ & 7 \\
\hline 29 & $\begin{array}{l}\text { Solche Vermählung ist ratificirt worden in dem } \\
\text { Consistorio der Allerheiligsten Dreyfaltigkeit/ }\end{array}$ & $\mathrm{e}$ & 1 & $\mathrm{n}$ & 0 & 1 & 0 & 1 & $\mathrm{r}$ & 3 \\
\hline $\begin{array}{l}16 / \\
12\end{array}$ & $\begin{array}{l}\text { und ertheilte derselben ein solchen Glantz der } \\
\text { Gnaden und Heiligkeit/ }\end{array}$ & $\mathrm{e}$ & 0 & $\mathrm{e}$ & 1 & 0 & 0 & 0 & $\mathrm{r}$ & 4 \\
\hline 18 & $\begin{array}{l}\text { wie er ihr möchte suchen (...) einen Mann/ welcher } \\
\text { das Haubt eines Weibs von dem Tarsensischen } \\
\text { Prediger Paolo genennt wird/ }\end{array}$ & $\mathrm{n}$ & 0 & $\mathrm{n}$ & 0 & 0 & 0 & 1 & $r$ & 2 \\
\hline 21 & $\begin{array}{l}\text { GOTT suchte demnach durch die gantze Zeit deß } \\
\text { Alten Testaments/ }\end{array}$ & $\mathrm{e}$ & 1 & $\mathrm{e}$ & 1 & 0 & 0 & 1 & $\mathrm{r}$ & 4 \\
\hline
\end{tabular}




\begin{tabular}{|l|l|c|c|c|c|c|c|c|c|c|}
\hline 11 & $\begin{array}{l}\text { dann weil die Allerheiligste Dreyfaltigkeit den ersten } \\
\text { und vollkommesten Ehestand in der Favorita deß } \\
\text { Paradeis hat eingestellt zwischen zwey gleichen } \\
\text { Personen/ }\end{array}$ & $\mathrm{n}$ & 0 & $\mathrm{n}$ & 0 & 0 & 0 & $/$ & $\mathrm{r}$ & 2 \\
\hline 19 & $\begin{array}{l}\text { weil Maria war ein Zier und Geschirr aller } \\
\text { Reinigkeit/ }\end{array}$ & e & 1 & e & 0 & 0 & 0 & $/$ & $\mathrm{r}$ & $\mathbf{4}$ \\
\hline 20 & $\begin{array}{l}\text { weil sie war Glantz und ein Krantz aller } \\
\text { Jungfrauschafft/ }\end{array}$ & e & 1 & e & 0 & 0 & 0 & $/$ & $\mathrm{r}$ & $\mathbf{4}$ \\
\hline
\end{tabular}

Tabelle 5: Matthias J. Scheeben /synthetischer Genitiv/

\begin{tabular}{|c|c|c|c|c|c|c|c|c|c|c|}
\hline $\mathrm{S}$. & Text & $\mathrm{A}$ & $\mathrm{B}$ & $\mathrm{C}$ & $\mathrm{D}$ & $\mathrm{E}$ & $\mathrm{F}$ & $\mathrm{G}$ & $\mathrm{H}$ & I \\
\hline $17 / \mathrm{T}$ & Das Mysterium der heiligsten Dreifaltigkeit. & $\mathrm{e}$ & 1 & $\mathrm{n}$ & 0 & 1 & 0 & $s$ & $\mathrm{r}$ & 3 \\
\hline $\mathbf{T}$ & Das Dunkel des Mysteriums. & $\mathrm{n}$ & 0 & $\mathrm{n}$ & 0 & 0 & 0 & $\mathrm{~s}$ & $\mathrm{r}$ & 2 \\
\hline 1 & $\begin{array}{l}\text { Wir beginnen mit dem anbetungswïrdigen } \\
\text { Mysterium der allerheiligsten Dreifaltigkeit. }\end{array}$ & $\mathrm{e}$ & 1 & e & 1 & 1 & 0 & 1 & $r$ & 4 \\
\hline 3 & $\begin{array}{l}\text { Das erste, was wir zu tun haben, besteht darin, daß } \\
\text { wir den geheimnisvollen Charakter dieser Lehre } \\
\text { beweisen und erklären. }\end{array}$ & $\mathrm{e}$ & 1 & $\mathrm{e}$ & 1 & 0 & 0 & 0 & $\mathrm{r}$ & 4 \\
\hline 4 & $\begin{array}{l}\text { Der Gedanke, die Dreifaltigkeit der Personen in } \\
\text { der einen göttlichen Natur durch Vernunftgründe } \mathrm{zu} \\
\text { beweisen, }\end{array}$ & $\mathrm{n}$ & 0 & $\mathrm{n}$ & 0 & 0 & 0 & 0 & 1 & 2 \\
\hline 12 & $\begin{array}{l}\text { In der neueren Zeit hat namentlich die Günthersche } \\
\text { Schule den Versuch gemacht, diese wie alle andern } \\
\text { Geheimnislehren des Christentums (...) aufzulösen. }\end{array}$ & $\mathrm{n}$ & 0 & $\mathrm{e}$ & 2 & 0 & 0 & 0 & 1 & 4 \\
\hline 14 & $\begin{array}{l}\text { Es bedarf jedoch nur einer einfachen Betrachtung } \\
\text { der Glaubenslehre, }\end{array}$ & $\mathrm{n}$ & 0 & $\mathrm{e}$ & 1 & 0 & 0 & 1 & $r$ & 3 \\
\hline 18 & $\begin{array}{l}\text { Das ist die einstimmige, entschiedene Ansicht fast } \\
\text { aller Theologen vom hl. Thomas herab bis nahe an } \\
\text { die neueste Zeit, }\end{array}$ & $\mathrm{e}$ & 2 & $\mathrm{e}$ & 2 & 0 & 0 & 1 & $r$ & 5 \\
\hline $18 / 9$ & $\begin{array}{l}\text { Wie die Natur selbst allen drei Personen } \\
\text { gemeinschaftlich ist, so ist es auch die göttliche } \\
\text { Wirksamkeit nach außen, welche nur durch die } \\
\text { Kraft der gemeinschaftlichen Natur ausgeübt wird. }\end{array}$ & $\mathrm{e}$ & 1 & $n$ & 0 & 0 & 0 & 1 & $r$ & 3 \\
\hline 10 & $\begin{array}{l}\text { Vater, Sohn und der heilige Geist sind ein Prinzip } \\
\text { aller Dinge: }\end{array}$ & $\mathrm{e}$ & 1 & $\mathrm{n}$ & 0 & 0 & 0 & 1 & $r$ & 3 \\
\hline 12 & $\begin{array}{l}\text { Daraus folgt, daß aus den Werken Gottes nach } \\
\text { außen ... }\end{array}$ & $\mathrm{n}$ & 0 & $\mathrm{n}$ & 0 & 0 & 0 & 1 & $r$ & 2 \\
\hline 15 & $\begin{array}{l}\text { Oder kann man aus den Werken Gottes auf etwas } \\
\text { anderes schließen, }\end{array}$ & $\mathrm{n}$ & 0 & $\mathrm{n}$ & 0 & 0 & 0 & 1 & $\mathrm{t}$ & 2 \\
\hline 19 & $\begin{array}{l}\text { kann man zur Erklärung der Schöpfung etwas } \\
\text { anderes fordern als ... }\end{array}$ & $\mathrm{n}$ & 0 & $\mathrm{n}$ & 0 & 0 & 0 & 1 & $\mathrm{t}$ & 2 \\
\hline 19 & $\begin{array}{l}\text {...die Existenz einer unendlich vollkommenen, } \\
\text { außer= und überweltlichen Kraft ? }\end{array}$ & $\mathrm{e}$ & 4 & $\mathrm{n}$ & 0 & 0 & 0 & 0 & $\mathrm{r}$ & 6 \\
\hline 25 & $\begin{array}{l}\text { Die Vernunft erkennt (...) den konkreten, } \\
\text { bestimmten, wirklichen Gott, weil nur der konkrete, } \\
\text { wirkliche Gott Schöpfer der Welt sein kann. }\end{array}$ & $\mathrm{n}$ & 0 & $\mathrm{n}$ & 0 & 0 & 0 & 1 & $r$ & 2 \\
\hline
\end{tabular}




\begin{tabular}{|c|c|c|c|c|c|c|c|c|c|c|}
\hline $19 / 6$ & $\begin{array}{l}\text { Allerdings sagt der Apostel: "Das Unsichtbare } \\
\text { Gottes wird in den geschaffenen Dingen geistig } \\
\text { erschaut." }\end{array}$ & $\mathrm{n}$ & 0 & $\mathrm{n}$ & 0 & 0 & 0 & $s$ & $\mathrm{r}$ & 2 \\
\hline 7 & Aber was ist dieses Unsichtbare Gottes? & $\mathrm{n}$ & 0 & $\mathrm{e}$ & 1 & 0 & 0 & 1 & $\mathrm{t}$ & 3 \\
\hline 9 & $\begin{array}{l}\text { Es ist }(. . .) \text { dasjenige, was in den Werken Gottes } \\
\text { hervortritt, }\end{array}$ & $\mathrm{n}$ & 0 & $\mathbf{n}$ & 0 & 0 & 0 & 1 & $\mathrm{r}$ & 2 \\
\hline 20 & $\begin{array}{l}\text { Darum sagt der hl. Johannes mit Recht (...) und noch } \\
\text { deutlicher der Sohn Gottes selbst: }\end{array}$ & $\mathrm{n}$ & 0 & $\mathrm{n}$ & 0 & 0 & 0 & $s$ & $\mathrm{r}$ & 2 \\
\hline 23 & $\begin{array}{l}\text { Nur die göttlichen Personen selbst, die in dem } \\
\text { unzugänglichen Lichte der Gottheit wohnen, } \\
\text { können also sich (...) erkennen; ... }\end{array}$ & $\mathrm{n}$ & 0 & $\mathrm{e}$ & 1 & 0 & 0 & 1 & $\mathrm{r}$ & 3 \\
\hline 26 & $\begin{array}{l}\text {...nur der Vater und der Sohn und der von beiden } \\
\text { ausgehende Geist, welcher "in Innern Gottes wohnt }\end{array}$ & $\mathrm{n}$ & 0 & $\mathrm{n}$ & 0 & 0 & 0 & 1 & $\mathrm{r}$ & 2 \\
\hline 27 & ...und die Tiefen der Gottheit erforscht", & $\mathrm{n}$ & 0 & $\mathrm{n}$ & 0 & 0 & 0 & 0 & $\mathrm{r}$ & 2 \\
\hline 30 & $\begin{array}{l}\text { nur sie erkennen einander aus sich; alle geschaffenen } \\
\text { Geister erkennen diese Personen bloß durch deren } \\
\text { gnädige Offenbarung, nicht auf dem Wege des } \\
\text { Schauens oder des Forschens, ... } \\
\end{array}$ & $\mathrm{e}$ & 0 & $\mathrm{n}$ & 0 & 0 & 0 & 1 & $\mathrm{r}$ & 3 \\
\hline 31 & $\begin{array}{l}\text {...sondern ausschließlich auf dem Wege der } \\
\text { Erfahrung ... }\end{array}$ & $\mathrm{n}$ & 0 & $\mathrm{n}$ & 0 & 0 & 0 & 1 & $r$ & 2 \\
\hline 31 & ....aus positiver Mitteilung Gottes. & $\mathrm{n}$ & 0 & $\mathrm{e}$ & 1 & 0 & 0 & 1 & $r$ & 3 \\
\hline 32 & $\begin{array}{l}\text { Die Vernunft sieht also ein, daß sie aus sich nicht zur } \\
\text { Erkenntnis der Dreifaltigkeit gelangen kann; }\end{array}$ & 0 & $\mathrm{n}$ & 0 & 0 & 0 & 1 & $r$ & 2 & \\
\hline 35 & $\begin{array}{l}\text { Die natïrliche Vernunft erkennt bloß die Natur } \\
\text { Gottes, ... }\end{array}$ & $n$ & 0 & $\mathrm{n}$ & 0 & 0 & 0 & 0 & $r$ & 2 \\
\hline 36 & $\begin{array}{l}\text {...und auch diese nur nach ihrer Außenseite, als die } \\
\text { höchste Ursache der geschaffenen Natur, ... }\end{array}$ & $\mathrm{e}$ & 1 & $\mathrm{e}$ & 1 & 1 & 0 & 1 & $r$ & 4 \\
\hline 37 & $\begin{array}{l}\text {...ohne in die innern Tiefen der Gottheit } \\
\text { eindringen zu können, ... }\end{array}$ & $\mathrm{n}$ & 0 & $\mathrm{e}$ & 1 & 0 & 0 & 1 & 1 & 3 \\
\hline $20 / 2$ & $\begin{array}{l}\text {...staunend und anbetend muß sie vor dem } \\
\text { undurchdringlichen Schleier, der das Angesicht } \\
\text { Gottes bedeckt, stehen bleiben, }\end{array}$ & $\mathrm{n}$ & 0 & $n$ & 0 & 0 & 0 & 0 & $r$ & 2 \\
\hline 5 & $\begin{array}{l}\text { bis er selbst sein Inneres aufschließt, um uns die } \\
\text { unbegreiflichen Geheimnisse desselben ... }\end{array}$ & $\mathrm{n}$ & 0 & $\overline{\mathrm{e}}$ & 1 & 0 & 0 & 0 & 1 & 3 \\
\hline 5 & jetzt im ahnungsvollen Dunkel des Glaubens, ... & $\mathrm{n}$ & 0 & $\mathrm{e}$ & 1 & 0 & 0 & 1 & 1 & 3 \\
\hline 5 & $\begin{array}{l}\text {...dereinst in der lichten Klarheit seiner } \\
\text { Anschaung zu zeigen. }\end{array}$ & $\mathrm{e}$ & 1 & e & 1 & 0 & 1 & I & I & 4 \\
\hline 13 & $\begin{array}{l}\text { In dem unendlichen Reichtum der göttlichen } \\
\text { Natur allein ist es möglich, }\end{array}$ & $\mathrm{e}$ & 1 & $\mathrm{e}$ & 1 & 0 & 0 & l & $\mathrm{t}$ & 4 \\
\hline 19 & $\begin{array}{l}\text { wir können ferner, aber nur mit der ganzen } \\
\text { Anstrengung der gläubigen Vernunft, ... }\end{array}$ & $\mathrm{e}$ & 1 & $\mathrm{e}$ & 1 & 0 & 0 & I & $\mathrm{r}$ & 4 \\
\hline 20 & ...die Inklonkludenz der Gründe, ... & $\mathrm{n}$ & 0 & $\mathrm{n}$ & 0 & 0 & 0 & 0 & $\mathrm{r}$ & 2 \\
\hline 21 & $\begin{array}{l}\text {...mit denen man einen Widerspruch im } \\
\text { Gegenstande unseres Glaubens dartun will, mit } \\
\text { Bestimmtheit nachweisen. }\end{array}$ & $\mathrm{e}$ & 1 & $\mathrm{n}$ & 0 & 0 & 1 & 1 & $r$ & 3 \\
\hline
\end{tabular}




\begin{tabular}{|l|l|c|c|c|c|c|c|c|c|c|}
\hline 23 & $\begin{array}{l}\text { Allein ohne die Offenbarung oder abgesehen von } \\
\text { ihr, haben wir nichts, was uns auch nur den } \\
\text { Gedanken an die Möglichkeit einer } \\
\text { Dreipersönlichkeit in Gott nahezulegen vermöchte. }\end{array}$ & $\mathrm{n}$ & 0 & $\mathrm{n}$ & 0 & 0 & 0 & $/$ & $\mathrm{r}$ & 2 \\
\hline 25 & $\begin{array}{l}\text { Man könnte mir antworten, auch bei den } \\
\text { Eigenschaften der göttlichen Natur, (...) können } \\
\text { wir (...) begreifen und beweisen, }\end{array}$ & e & 1 & $\mathrm{n}$ & 0 & 0 & 0 & $/$ & $\mathrm{r}$ & 3 \\
\hline 30 & $\begin{array}{l}\text { Allein zuerst ist die Unbegreiflichkeit dieser } \\
\text { Gegenstände nicht so tief }\end{array}$ & e & 1 & $\mathrm{n}$ & 0 & 0 & 0 & $\mathrm{~s}$ & $\mathrm{r}$ & 3 \\
\hline 34 & $\begin{array}{l}\text { Dann aber (...) haben wir in unserer Vernunft } \\
\text { Gründe, welche uns zwingen, die Wirklichkeit } \\
\text { dieser Gegenstände und damit auch die } \\
\text { Möglichkeit anzunehmen. }\end{array}$ & e & 1 & $\mathrm{n}$ & 0 & 0 & 0 & $\mathrm{o}$ & $\mathrm{r}$ & 3 \\
\hline 36 & $\begin{array}{l}\text { Gott muß allgegenwärtig, ewig, frei sein, weil er } \\
\text { sonst nicht die schöpferische Ursache der Welt } \\
\text { sein könnte. }\end{array}$ & $\mathrm{n}$ & 0 & $\mathrm{e}$ & 1 & 0 & 0 & $/$ & $\mathrm{r}$ & 3 \\
\hline 37 & $\begin{array}{l}\text { Haben wir aber auch in unserer Vernunft einen } \\
\text { notwendigen Grund, die Wirklichkeit der } \\
\text { Dreifaltigkeit anzunehmen? }\end{array}$ & $\mathrm{n}$ & 0 & $\mathrm{n}$ & 0 & 0 & 0 & $\mathrm{o}$ & $/$ & 2 \\
\hline $21 / \mathrm{T}$ & $\begin{array}{l}\text { Kritik der Versuche, es mit der bloßen Vernunft } \\
\text { nachzuweisen. }\end{array}$ & $\mathrm{n}$ & 0 & $\mathrm{n}$ & 0 & 0 & 0 & $\mathrm{~s}$ & $\mathrm{r}$ & 2 \\
\hline 1 & $\begin{array}{l}\text { Die Dreifaltigkeit der Personen ist in Gott objektiv } \\
\text { notwendig; }\end{array}$ & $\mathrm{n}$ & 0 & $\mathrm{n}$ & 0 & 0 & 0 & $\mathrm{~s}$ & $\mathrm{t}$ & 2 \\
\hline 12 & $\begin{array}{l}\text { Wir halten uns bloß an diejenigen, welche auch das } \\
\text { gesunde Auge eines aufrichtig gläubigen Christen } \\
\text { zu bestechen im stande sind ... }\end{array}$ & e & 2 & e & 1 & 0 & 0 & $\mathrm{o}$ & $/$ & 5 \\
\hline
\end{tabular}

Tabelle 7: Matthias J. Scheeben /analytischer Genitiv/

\begin{tabular}{|l|l|c|c|c|c|c|c|c|c|c|}
\hline S. & Text & A & B & C & D & E & F & G & H & I \\
\hline $21 / 8$ & Wir übergeben eine Menge von Beweisen, & $\mathrm{n}$ & 0 & $\mathrm{n}$ & 0 & 0 & 0 & o & r & 3 \\
\hline $22 / 8$ & $\begin{array}{l}\text { Einige fügen hinzu, das Leben sei ohne gewisse } \\
\text { reale Mannigfaltigkeit von Momenten, }\end{array}$ & $\mathrm{n}$ & 0 & $\mathrm{e}$ & 2 & 0 & 0 & $/$ & $\mathrm{r}$ & 5 \\
\hline $23 / 4$ & $\begin{array}{l}\text { Das gilt auch allen denjenigen, welche behaupten, } \\
\text { man könne gar keinen richtigen und wahren } \\
\text { Begriff von dem wirklichen Gotte haben, }\end{array}$ & $\mathrm{e}$ & 1 & $\mathrm{e}$ & 2 & 0 & 0 & $\mathrm{o}$ & $\mathrm{r}$ & 6 \\
\hline $\begin{array}{l}24 / \\
17\end{array}$ & $\begin{array}{l}\text { Demnach würde also das Argument unserer Gegner } \\
\text { nicht bloß eine Trinität, sondern eine Quinternität, } \\
\text { eine unendliche Zahl von Personen in Gott } \\
\text { beweisen. }\end{array}$ & $\mathrm{n}$ & 0 & $\mathrm{e}$ & 1 & 0 & 0 & $\mathrm{o}$ & $\mathrm{r}$ & 4 \\
\hline $\begin{array}{l}25 / \\
30\end{array}$ & $\begin{array}{l}\text { und dann argumentiert er auf dieselbe Weise wie } \\
\text { Anselmus weiter, um die ganze Lehre von der } \\
\text { Trinität zu entwickeln. }\end{array}$ & $\mathrm{n}$ & 0 & $\mathrm{e}$ & 1 & 0 & 0 & 0 & $/$ & 4 \\
\hline $\begin{array}{l}33 / \\
13\end{array}$ & $\begin{array}{l}\text { Unser (...) Beweis (...) hat sich (...) entschieden } \\
\text { bewährt, indem alle diese Verfahren, um zum Ziele } \\
\text { zu kommen, entweder irrationell werden müssen } \\
\text { durch Fehlschlüsse oder überrationell durch } \\
\text { Hinzunahme von Glaubenswahrheiten. }\end{array}$ & $\mathrm{n}$ & 0 & $\mathrm{n}$ & 0 & 0 & 0 & $/$ & $\mathrm{r}$ & 3 \\
\hline
\end{tabular}




\begin{tabular}{|c|c|c|c|c|c|c|c|c|c|c|}
\hline 40 & \begin{tabular}{|l|} 
und da ist es denn ganz natiurlich, daß unsere \\
Vorstellungen und Begriffe von der Trinität ...
\end{tabular} & $\mathrm{n}$ & 0 & $\mathrm{e}$ & 1 & 0 & 1 & $\mathrm{~s}$ & $r$ & 5 \\
\hline 2 & $\begin{array}{l}\text {...als die Begriffe von der göttlichen Natur und } \\
\text { ihren Eigenschaften. }\end{array}$ & e & 1 & $\mathrm{n}$ & 0 & 0 & 0 & $\mathbf{s}$ & $\mathrm{r}$ & 4 \\
\hline 14 & $\begin{array}{l}\text { Es ist klar, daß die Lehre von der Trinität (...) } \\
\text { besitzt, }\end{array}$ & $n$ & 0 & $\mathrm{n}$ & 0 & 0 & 0 & $\mathrm{~s}$ & $\mathrm{r}$ & 3 \\
\hline $\begin{array}{l}39 / \\
15\end{array}$ & $\begin{array}{l}\text { Wir sagen: ein einziges Prinzip aus der } \\
\text { Glaubenslehre von der Trinität genügt der } \\
\text { Vernunft, }\end{array}$ & $\mathrm{n}$ & 0 & $\mathrm{n}$ & 0 & 0 & 0 & $\mathrm{~s}$ & $r$ & 3 \\
\hline $40 / 8$ & $\begin{array}{l}\text { Bei den beiden produzierten Personen ist das } \\
\text { offenbar, da sie die Natur nur von der ersten } \\
\text { haben, }\end{array}$ & $\mathrm{n}$ & 0 & $\mathrm{n}$ & 0 & 0 & 0 & 0 & $r$ & 3 \\
\hline $42 / 6$ & $\begin{array}{l}\text { Diese Relationen aber müssen Personalrelationen, } \\
\text { Relationen von Personen sein; }\end{array}$ & $\mathrm{n}$ & 0 & $\mathrm{n}$ & 0 & 0 & 0 & 1 & $\mathrm{r}$ & 3 \\
\hline 17 & $\begin{array}{l}\text { d.h. sie begründen eine Vielheit von Personen in } \\
\text { derselben Natur. }\end{array}$ & $\mathrm{n}$ & 0 & $\mathrm{n}$ & 0 & 0 & 0 & 0 & $r$ & 3 \\
\hline 30 & $\begin{array}{l}\text { Vermittels des Lehrfaßes von den Produktionen } \\
\text { wird also die Gotteserkenntnis gerade auf dem } \\
\text { Punkte angegriffen und fortgeführt, }\end{array}$ & $\mathrm{n}$ & 0 & $\mathrm{n}$ & 0 & 0 & 0 & 1 & $\mathrm{t}$ & 3 \\
\hline $43 / 6$ & $\begin{array}{l}\text { während er bis dahin - die Lehre von der } \\
\text { Prädestination allein ausgenommen, (...) - alles, } \\
\text { (...) als Vernunftwahrheiten betrachtete und } \\
\text { behandelte. }\end{array}$ & $\mathrm{n}$ & 0 & $\mathrm{n}$ & 0 & 0 & 0 & $\mathrm{~s}$ & 1 & 3 \\
\hline 10 & $\begin{array}{l}\text { Dieser Glaubensfaß ist aber gerade der von den } \\
\text { beiden Prozessionen und Produktionen. }\end{array}$ & $\mathrm{e}$ & 1 & $\mathrm{n}$ & 0 & 0 & 0 & 1 & $\mathbf{r}$ & 5 \\
\hline $45 / 6$ & $\begin{array}{l}\text { Die Vernunft gibt uns nicht nur den Begriff von der } \\
\text { göttlichen Natur,... }\end{array}$ & $\mathrm{e}$ & 1 & $\mathrm{n}$ & 0 & 0 & 0 & 0 & $\mathrm{r}$ & 4 \\
\hline 7 & $\begin{array}{l}\text { uuch (den Begriff) von ihrer } \\
\text { len Lebendigkeit. }\end{array}$ & $\mathrm{e}$ & 2 & $\mathrm{n}$ & 0 & 0 & 1 & o & $\mathbf{r}$ & 4 \\
\hline $47 / 6$ & $\begin{array}{l}\text { Das Erkennen denken wir uns nämlich als das } \\
\text { Erzeugen einer Vorstellung von einer Sache }\end{array}$ & $\mathrm{n}$ & 0 & $\mathrm{n}$ & 0 & 0 & 0 & 1 & $r$ & 3 \\
\hline $48 / 2$ & $\begin{array}{l}\text { man kann zwischen dem Sein Gottes und der } \\
\text { Vorstellung von demselben im Erkenntnisakte } \\
\text { keinen reellen Unterschied machen. } \\
\end{array}$ & $\mathrm{n}$ & 0 & $\mathrm{n}$ & 0 & 0 & 0 & 1 & $r$ & 3 \\
\hline 17 & $\begin{array}{l}\text { Folglich, so urteilt die Vernunft, fällt jene Art von } \\
\text { Produktion, (...) weg; }\end{array}$ & $\mathrm{n}$ & 0 & $\mathrm{e}$ & 1 & 0 & 0 & $\mathrm{~s}$ & $\mathrm{r}$ & 3 \\
\hline $55 / 6$ & $\begin{array}{l}\text { Es gibt also in Gott außer der ersten Person, die sich } \\
\text { auf keine andere frühere bezieht, noch zwei andere } \\
\text { Weisen von Substanz oder Selbstständigkeit, }\end{array}$ & $\mathrm{e}$ & 0 & $\mathrm{e}$ & 2 & 0 & 0 & 1 & $r$ & 6 \\
\hline $\begin{array}{l}56 / \\
21\end{array}$ & $\begin{array}{l}\text { So ist ein Teil unseres Körpers, z.B. der Kopf, } \\
\text { obgleich selbst Träger von Eigenschaften und } \\
\text { Akzidenzien, }\end{array}$ & $\mathrm{e}$ & 0 & $\mathrm{n}$ & 0 & 0 & 0 & 1 & $r$ & 4 \\
\hline \begin{tabular}{|l|}
$57 /$ \\
31 \\
\end{tabular} & $\begin{array}{l}\text { das Tier (...) aber es hat kein eigentliches Recht auf } \\
\text { dieselben, keinen bewußten Genuß von denselben, }\end{array}$ & $\mathrm{n}$ & 0 & $\mathrm{e}$ & 1 & 0 & 0 & 0 & $\mathrm{r}$ & 4 \\
\hline $\begin{array}{l}61 / \\
29\end{array}$ & $\begin{array}{l}\text { Der Hauch der göttlichen Liebe ist also ein } \\
\text { persönlicher Hauch, eine Person und eine von den } \\
\text { durch ihre Liebe ihn aushauchenden Personen } \\
\text { verschiedene Person; }\end{array}$ & $\mathrm{e}$ & 1 & $\mathrm{n}$ & 0 & 0 & 0 & 1 & $r$ & 4 \\
\hline
\end{tabular}




\begin{tabular}{|c|c|c|c|c|c|c|c|c|c|c|}
\hline $\begin{array}{l}66 / \\
20\end{array}$ & $\begin{array}{l}\text { Daher können sie auch dann voneinander } \\
\text { verschieden und pesönlich gegeneinader selbständig } \\
\text { sein, wenn keine von der andern ihren Ursprung hat }\end{array}$ & $\mathrm{n}$ & 0 & $\mathrm{n}$ & 0 & 0 & 0 & $\mathrm{~s}$ & $\mathrm{r}$ & 3 \\
\hline $\begin{array}{l}73 / \\
14\end{array}$ & $\begin{array}{l}\text { Vielmehr scheint die Grundform von ersterem } \\
\text { (Wurzel: tuh, ducere) ... }\end{array}$ & $\mathrm{n}$ & 0 & $\mathrm{n}$ & 0 & 0 & 0 & $\mathrm{~s}$ & $\mathrm{t}$ & 3 \\
\hline 14 & ...die Bedeutung von ziehen, ... & $\mathrm{n}$ & 0 & $\mathrm{n}$ & 0 & 0 & 0 & 0 & $\mathrm{r}$ & 3 \\
\hline 15 & ... (...) die Bedeutung von zeigen zu haben. & $\mathrm{n}$ & 0 & $\mathrm{n}$ & 0 & 0 & 0 & 0 & $r$ & 3 \\
\hline 26 & $\begin{array}{l}\text { Und in der Tat legt auch das zeugende Wesen in der } \\
\text { sinnlichen Welt nicht so sehr Zeugnis ab von seiner } \\
\text { konkreten, individuellen Natur,... }\end{array}$ & $\mathrm{e}$ & 3 & n & 0 & 0 & 1 & 1 & $\mathrm{r}$ & 6 \\
\hline $\begin{array}{l}79 / \\
14 \\
\end{array}$ & Sie ist nur Geist vom Geiste. & $\mathrm{n}$ & 0 & $\mathrm{n}$ & 0 & 0 & 0 & 1 & r & 3 \\
\hline 15 & $\begin{array}{l}\text { "Der heilige Geist ist von beiden als Geist vom } \\
\text { Geiste: denn Gott ist Geist." }\end{array}$ & $\mathbf{n}$ & 0 & $\mathrm{n}$ & 0 & 0 & 0 & 1 & & 3 \\
\hline $83 / 9$ & $\begin{array}{l}\text { so erscheint er sofort als ein Träger des Lebens, (...) } \\
\text { ebenso wie der Sohn durch die Einheit von Wort } \\
\text { und Bild als Erzeugter (...) erscheint. }\end{array}$ & $\mathrm{e}$ & 0 & $\mathrm{n}$ & 0 & 0 & 0 & 1 & $r$ & 4 \\
\hline $85 / 9$ & $\begin{array}{l}\text { Wir brauchen nun nicht mehr zu sagen, (...); wir } \\
\text { haben einen ganz bestimmten, spezifischen und } \\
\text { charakteristischen Namen von der tiefsten } \\
\text { Bedeutung. }\end{array}$ & $\mathrm{e}$ & 1 & e & 4 & 1 & 0 & 0 & $\mathrm{r}$ & 8 \\
\hline $\begin{array}{l}89 / \\
16 \\
\end{array}$ & $\begin{array}{l}\text { In jedem Akte der Liebe (...) findet sich übrigens } \\
\text { eine gewisse Art von Freiheit, }\end{array}$ & $\mathrm{n}$ & 0 & $\mathrm{e}$ & 1 & 0 & 0 & $\mathbf{S}$ & $r$ & 4 \\
\hline $\begin{array}{l}90 / \\
20\end{array}$ & $\begin{array}{l}\text { Wenngleich der Ausdruck sehr leicht zu } \\
\text { Mißverständnissen führen kann, so hat er doch auch } \\
\text { anderseits einen so tiefen Kern von Wahrheit, }\end{array}$ & $\mathrm{n}$ & 0 & $\mathrm{e}$ & 1 & 0 & 0 & 0 & $\mathrm{r}$ & 4 \\
\hline $\begin{array}{l}94 / \\
13\end{array}$ & $\begin{array}{l}\text { erscheint er uns wie ein unendlich kostbarer, aus } \\
\text { ihrem Liebes- und Lebenshauche kristallisierter } \\
\text { Diamant von unerschütterlicher Festigkeit und } \\
\text { der lautersten Reinheit, }\end{array}$ & $\mathrm{e}$ & 2 & $\mathrm{e}$ & 3 & 1 & 0 & 1 & $\mathrm{r}$ & 9 \\
\hline $\begin{array}{l}95 / \\
11\end{array}$ & $\begin{array}{l}\text { so ist doch ihre Analogie so reich, (...), daß ihr } \\
\text { Verständnis uns die klarste und anschaulichste } \\
\text { Vorstellung von dem erhabenen Mysterium gibt, }\end{array}$ & 1 & 1 & $\mathrm{e}$ & 2 & 2 & 0 & 0 & $\mathrm{r}$ & 6 \\
\hline 27 & $\begin{array}{l}\text { sie setzt die Zeugung auch wesentlich voraus, und in } \\
\text { eben dieser ihrer positiven Beziehung zur Zeugung } \\
\text { liegt zugleich ihr Unterschied von derselben. }\end{array}$ & $\mathrm{n}$ & 0 & $\mathrm{e}$ & 1 & 0 & 1 & $s$ & $r$ & 4 \\
\hline $\begin{array}{l}97 / \\
10\end{array}$ & $\begin{array}{l}\text { Wie die Mutter das Liebesband zwischen Vater und } \\
\text { Kind ist, (...), indem sie dem Kinde die Natur. vom } \\
\text { Vater ubermittelt, }\end{array}$ & $\mathrm{n}$ & 0 & $\mathrm{n}$ & 0 & 0 & 0 & 0 & $r$ & 3 \\
\hline $\begin{array}{l}99 / \\
36\end{array}$ & $\begin{array}{l}\text { Weil sie von Vater und Sohn genommen ist, (...), } \\
\text { muß auch sie Geist genannt werden, als Geist vom } \\
\text { Geiste, }\end{array}$ & $\mathrm{n}$ & 0 & $\mathrm{n}$ & 0 & 0 & 0 & 1 & $\mathrm{r}$ & 3 \\
\hline $\begin{array}{l}100 / \\
2\end{array}$ & $\begin{array}{l}\text { so deutet der Name "Geist" als Eigenname der } \\
\text { dritten Person, also im Sinne von spiramen, } \\
\text { wesentlich hin auf den Namen Geist, }\end{array}$ & $\mathrm{n}$ & 0 & $\mathrm{n}$ & 0 & 0 & 0 & 1 & $\mathrm{r}$ & 3 \\
\hline $\begin{array}{l}102 / \\
24\end{array}$ & $\begin{array}{l}\text { Wir glauben gezeigt zu haben, wie sich die } \\
\text { geheimnisvolle Lehre von der göttlichen } \\
\text { Dreifaltigkeit (...) entwickeln (...) läßt. }\end{array}$ & $\mathrm{e}$ & 1 & $\mathrm{e}$ & 1 & 0 & 0 & $\mathbf{S}$ & $\mathrm{r}$ & 5 \\
\hline $\begin{array}{l}105 / \\
\mathrm{T}\end{array}$ & $\begin{array}{l}\text { Die Vereinigung von Licht und Dunkelheit in der } \\
\text { Erkenntnis des Mysteriums. }\end{array}$ & e & 0 & $\mathrm{n}$ & 0 & 0 & 0 & $\mathrm{~s}$ & $r$ & 4 \\
\hline
\end{tabular}




\begin{tabular}{|l|l|c|c|c|c|c|c|c|c|c|}
\hline 5 & $\begin{array}{l}\text { daß wir einen evidenten inneren Wiederspruch darin } \\
\text { finden würden, wenn einer von ihnen nicht mit dem } \\
\text { übrigen auf den Gegenstand angewendet werden } \\
\text { Sollte. }\end{array}$ & $\mathrm{n}$ & 0 & $\mathrm{n}$ & 0 & 0 & 0 & $\mathrm{~s}$ & $\mathrm{t}$ & 3 \\
\hline $\begin{array}{l}109 / \\
5\end{array}$ & $\begin{array}{l}\text { desto weniger werden wir versucht sein, eine } \\
\text { Unvereinbarkeit seiner einzelner Momente } \\
\text { anzunehmen und aus der unerfaßlichen Erhabenheit } \\
\text { desselben ein düsteres Gewirre von } \\
\text { Wiedersprüchen zu machen. }\end{array}$ & $\mathrm{n}$ & 0 & $\mathrm{n}$ & 1 & 0 & 0 & $\mathrm{o}$ & $/$ & 4 \\
\hline 20 & $\begin{array}{l}\text { Man spricht heutzutage viel von der philosophischen } \\
\text { Bedeutung des Dogmas von der göttlichen Trinität. }\end{array}$ & $\mathrm{e}$ & 1 & $\mathrm{n}$ & 0 & 0 & 0 & $/$ & $\mathrm{r}$ & 4 \\
\hline $\begin{array}{l}110 / \\
14\end{array}$ & $\begin{array}{l}\text { Zu diesen Wahrheiten gehört die Lehre von der } \\
\text { Trinität in keiner Weise. }\end{array}$ & $\mathrm{n}$ & 0 & $\mathrm{n}$ & 0 & 0 & 0 & $\mathrm{~s}$ & $\mathrm{r}$ & 3 \\
\hline
\end{tabular}

\section{Statistische Resultate und Interpretation}

Die Vorkommenswahrscheinlichkeit des synthetischen und des analytischen Genitivs in älteren Texten:

\begin{tabular}{|l|c|c|c|c|}
\hline Frequenz der Belege & Der ahd.Isidor & $\begin{array}{c}\text { Meister } \\
\text { Eckhart }\end{array}$ & Martin Luther & $\begin{array}{c}\text { Abraham a } \\
\text { Sancta Clara }\end{array}$ \\
\hline NGr mit synthet. Genitiv & 110 & 191 & 236 & 446 \\
\hline NGr mit analyt. Genitiv & 1 & 8 & 13 & 10 \\
\hline
\end{tabular}

Der synthetische Genitiv kommt in allen untersuchten Texten viel häufiger vor als der analytische. Die Vorkommenshäufigkeit des analytischen Genitivs unterstützt die Vorhersage, daß verstärkte Konstruktionen in Texten seltener vorkommen als geschwächte Varianten.

Der Unterschied in der Gebrauchsfrequenz zwischen beider Konstruktionen in diesen älteren Texten ist sehr gering. Wegen dieser kleinen Gebrauchsfrequenz des analytischen Genitivs wäre eine größere Datenbasis notwendig, um die Grammatikalisierung der verstärkten Variante genauer beschreiben zu können.

Anschließend haben wir auch nach Belegen beider Konstruktionen in einem jüngeren Text gesucht. In der theologischen Schrift von Matthias Scheeben aus den sechziger Jahren des 19.Jahrhunderts waren genügend Belege von beiden Genitivformen zu finden. Der synthetische Genitiv kommt auch in diesem Text häufiger vor (im Durchschnitt 7,7 Belege auf eine Seite im Text) als der analytische (im Durchschnitt 0,56 Belege auf eine Seite im Text). Die Zahl der Belege des analytischen Genitivs (285 Belege auf 512 Seiten der Erstausgabe) als auch die Summe der Belege des synthetischen Genitivs (284 Belege auf 37 Seiten der Erstausgabe) ermöglichten den Vergleich der unmittelbaren grammatischen Umgebung bzw. der Verhältnisse, in denen beide Varianten vorkommen. Dabei sind wir von der Annahme ausgegangen, da $\beta$ 
der analytische Genitiv in komplizierteren bzw. markierteren grammatischen Verhältnissen vorkommt, da diese Variante auch im Gegenwartsdeutschen als verstärkte Konstruktion anzusehen ist.

Die folgende Tabelle zeigt die Anteile der markierten Parameterwerte der unmittelbaren grammatischen Umgebung des synthetischen und analytischen Genitivs im Text von M.Scheeben:

\begin{tabular}{|l|c|c|c|}
\hline Matthias Scheeben & synthet.Genitiv & analyt.Genitiv & $\mathrm{Id}^{7}$ \\
\hline $\begin{array}{l}\text { A/synthet.Genitiv/analyt.Genitiv - erweitert } \\
\text { durch: Attribute, weitere Genitive, Dative } \\
(\%)\end{array}$ & 44,01 & 47,02 & 2,5 \\
\hline B/Attribute - Durchschnitt & 0,55 & 0,57 & $/$ \\
\hline C/Kern - erweitert (\%) & 29,23 & 36,84 & 2,5 \\
\hline D/Attribute - Durchschnitt & 0,30 & 0,48 & $/$ \\
\hline E/Komparative, Superlative (\%) & 3,52 & 8,07 & 2 \\
\hline F/ohne pronominale Elemente (\%) & 93,31 & 91,23 & -1 \\
\hline G/Akkusativobjekt (\%) & 27,46 & 42,81 & 4 \\
\hline H/Rhema (\%) & 89,79 & 94,39 & 1 \\
\hline I/flektierbare Wörter - Durchschnitt & 2,85 & 3,75 & $/$ \\
\hline
\end{tabular}

Die Markiertheitswerte sind in Prozenten (A,C,E,F,G,H) bzw. Durchschnittswerten (B,D,I) ausgedrückt. Um die Vorhersage, daß der analytische Genitiv die verstärkte Konstruktion ist, zu überprüfen, haben wir Werte der grammatischen Umgebung beider Varianten verglichen und dabei nur statistisch signifikante Ergebnisse berücksichtigt. Die statistische Signifikanz ist mittels des sogenannten Differenzindexes (Pavlić 1985, nach Petrič 1990, Teržan 1990) berechnet worden und zwar bei den Parametern, die in Prozenten ausgedrückt sind. Der Unterschied zwischen beiden Samples (in diesem Falle synthetischer vs. analytischer Genitiv) ist statistisch signifikant, wenn der Wert von Id $>2$ ist.

Die markierteren Parameterwerte der unmittelbaren grammatischen Umgebung des analytischen Genitivs zeigen höhere Anteile bzw. Durchschnittszahlen bei acht von neun Parametern, allerdings sind nur vier Unterschiede zwischen den Samples statistisch signifikant, was sich z.T. auf die zu geringe Stichprobengröße zurückführen läßt. Die Differenz zwischen beiden Konstruktionen, die die erweiterten Formen des synthetischen und analytischen Genitivs und des Kerns betrifft, beträgt 3,01\% (A) und 7,61\% (C), beide Vergleiche sind statistisch signifikant. Der Durchschnittswert der Attribute (B,D) des analytischen Genitivs ist größer als beim synthetischen und das betrifft beide Teile der Nominalgruppe. Was die Komparative und Superlative (E) angeht, haben wir Prozentwerte ermittelt, die kompliziertere Verhältnisse im Umfeld des analytischen Genitivs ausweisen (3,52\% gegenüber 8,02\%; der Unterschied beträgt

$7 \quad$ Id $=$ Differenzindex 
4,55\% und ist statistisch signifikant). Bei dem Parameter Satzglied (G) (Subjekt vs. Akkusativobjekt) ist der Unterschied zugunsten des analytischen Genitivs relativ groß, denn er beträgt sogar 15,35\%; der Vergleich ist statistisch signifikant. Der analytische Genitiv kommt auch öfter im Rhema des Satzes $(\mathrm{H})$ vor (der Unterschied im Vergleich zu dem synthetischen beträgt $4,6 \%$, ist aber nicht statistisch signifikant). Der letzte Parameterwert (I) zeigt auch eine für unsere Vorhersage günstige Situation, denn die Durchschnittszahl der flektierbaren Wörter ist im Falle des analytischen Genitivs größer.

Die angeführten statistisch signifikanten Unterschiede legen unserer Meinung nach nahe, den analytischen Genitiv als verstärkte Variante anzusehen. Seine grammatische Umgebung hat sich im Text von M.Scheeben als die markiertere erwiesen. Bei einem Parameter ergab sich keine statistische Signifikanz (Rhema) und bei einem sind die Resultate des Vergleiches für unsere Vorhersage nicht günstig (ohne pronominale Elemente). Die Belege stammen aus einem Text, sie sind von einem Autor geschrieben worden und auch das Thema ist einheitlich, so ist auszuschließen, daß die Gründe für die Markiertheit des analytischen Genitivs bei der Textsorte, aussersprachlicher Motivation oder dem Stil des Autors oder der Zeit zu suchen wären, denn der analytische Genitiv ersetzt in dem Text von M.Scheeben alle Genitivarten bzw. Situationen im Rahmen einzelner Arten, die in der Fachliteratur beschrieben werden. So kann auch die Möglichkeit ausgeschlossen werden, daß der Gebrauch in Form weniger Genitivarten oder sogar einer Genitivart eingeschränkt ist. Eine solche Einschränkung würde die Annahme von dem analytischen Genitiv als einer verstärkten Konstruktion bezeugen, es würde aber auch die Annahme naheliegen, daß der Text als solcher markiert ist und von den allgemeinen Befunden abweicht.

Die Grundannahme der slowenischen Schule der natürlichen Syntax sieht das eventuelle spätere Vorkommen der geschwächten Konstruktion unter weniger einfachen grammatischen Verhältnissen vor, was die Zunahme aller markierten Werte bestimmter Parameter widerspiegelt. Auf der anderen Seite kann die verstärkte Konstruktion in späteren Entwicklungsperioden unter weniger komplizierten grammatischen Verhältnissen vorkommen. Wegen der geringen Zahl der Belege des analytischen Genitivs in den älteren Entwicklungsstufen wäre der Vergleich der unmittelbaren grammatischen Umgebung dieser Struktur in den verschiedenen Jahrhunderten nicht statistisch signifikant. Die ausreichende Zahl der Belege der geschwächten Konstruktion, d.h. des synthetischen Genitivs ermöglicht dagegen einen Vergleich des Umfeldes dieser Struktur. In der unten folgenden Tabelle sind die markierteren Parameterwerte in Prozenten und Durchschnittszahlen ausgedrückt. Die statistische Signifikanz der Unterschiede zwischen den Stichproben ist aus dem Differenzindex ersichtlich. Gemäß der Grundannahme über den Wandel der grammatischen Umgebung einer geschwächten Konstruktion wurde erwartet, daß der synthetische Genitiv in den einander chronologisch folgenden Stichproben eine Tendenz zur Behauptung in markierterer Umgebung zeigt. Um das zu überzuprüfen, haben wir in allen Fällen den jüngeren Text mit dem älteren verglichen. ${ }^{8}$ 


\begin{tabular}{|l|c|c|c|c|c|}
\hline synthetischer Genitiv & Isidor & Eckhart & M.Luther & S.Clara & Scheeben \\
\hline $\begin{array}{l}\text { A/synthetischer Genitiv - erweitert durch: } \\
\text { Attribute/ weitere Genitive (\%) }\end{array}$ & 30 & 22,51 & $\mathbf{3 5 , 1 7}$ & $\mathbf{4 5 , 9 6}$ & 44,01 \\
\hline B/Attribute - Durchschnitt & 0,31 & 0,25 & $\mathbf{0 , 3 6}$ & $\mathbf{0 , 6 4}$ & 0,55 \\
\hline C/Kern - erweitert (\%) & 13,36 & 23,04 & $\mathbf{3 0 , 5 1}$ & 30,49 & 29,23 \\
\hline D/Attribute - Durchschnitt & 0,16 & 0,16 & $\mathbf{0 , 2 7}$ & $\mathbf{0 , 2 9}$ & $\mathbf{0 , 3 0}$ \\
\hline E/Komparative, Superlative (\%) & 0 & $\mathbf{2 , 6 2}$ & 1,69 & $\mathbf{7 , 4 0}$ & 3,52 \\
\hline F/ohne pronominale Elemente (\%) & 94,55 & 95,3 & 89,41 & 88,57 & 93,31 \\
\hline G/Akkusativobjekt (\%) & 21,82 & 16,23 & $\mathbf{2 9 , 6 6}$ & 26,46 & 27,46 \\
\hline H/Rhema (\%) & 65,45 & $\mathbf{8 4 , 8 2}$ & $\mathbf{9 0 , 6 8}$ & 92,15 & 89,79 \\
\hline I/flektierbare Wörter - Durchschnitt & 2,44 & $\mathbf{2 , 5 5}$ & $\mathbf{2 , 8 7}$ & $\mathbf{2 , 9 6}$ & 2,85 \\
\hline
\end{tabular}

Die Beobachtungen der Anteile der verschiedenen Parameterwerte des synthetischen Genitivs in verschiedenen Texten aus verschiedenen sprachgeschichtlichen Perioden zeigen die Veränderungen des Umfeldes der synthetischen Form.

Im Althochdeutschen (Der althochdeutsche Isidor) kommt der synthetische Genitiv in einem relativ schwach markierten Umfeld vor. Die Prozentwerte liegen alle (außer beim Parameter Rhema $(\mathrm{H})$ ) unter 50\%, in den Belegen finden wir z.B. keine Komparative und Superlative (E). Der Text aus dem Mittelhochdeutschen (Meister Eckhart) zeigt keine größeren Veränderungen im Vergleich zum Althochdeutschen Isidor. Die Prozentwerte der erweiterten Kerne (C) sind jedoch höher und zwar um 9,68\% (keine statistische Signifikanz). Der Anteil der Komparative und Superlative (E) ist ebenfalls höher (der Unterschied beträgt $2,62 \%$ und ist statistisch signifikant), ebenso der Anteil der Belege ohne pronominalisierte Formen (F) (der Unterschied beträgt $0,75 \%$; keine statistische Signifikanz). Die Konstruktion kommt öfter im Rhema des Satzes $(\mathrm{H})$ vor (der Unterschied beträgt $19,37 \%$ und ist statistisch signifikant), auch die Durchschnittszahl der flektierbaren Wörter (I) ist größer. Das Frühneuhochdeutsche ist die Periode in der Geschichte der deutschen Sprache, für die die Zunahme im Gebrauch der analytischen Konstruktionen typisch ist, wenn wir die Situation mit derjenigen im Mittelhochdeutschen und Althochdeutschen vergleichen. Falls wir voraussetzen können, daß das Vordringen einer analytischen Konstruktion den Rückzug der synthetischen (wenn beide füreinander ersetzbar sind) impliziert und daß sich dieser Rückzug als Verschiebung in ein weniger einfaches grammatisches Umfeld manifestiert, dann könnten das die Prozentwerte und Durchschnittszahlen des Vergleiches synthetischer Genitiv im Mittelhochdeutschen vs. synthetischer Genitiv im Frühneuhochdeutschen bestätigen. Der Prozentwert des erweiterten Genitivs (A) ist bei Luther höher, und zwar um 12,66\% (der Unterschied ist statistisch signifikant), ebenso der Prozentwert des erweiterten Kerns (C) (auch statistisch signifikant, der Unterschied beträgt 7,47\%). Die Durchschnittszahlen der

8 Fettgedruckte Zahlen stehen für Vergleiche, die zugleich hypothesenfreundlich und statistisch signifikant sind. 
Attribute im Genitivattribut (B), im Kern (D) und die der flektierbaren Wörter (I) sind höher. Der synthetische Genitiv kommt häufiger als Akkusativobjekt (G) (der Unterschied beträgt $13,43 \%$ und ist statistisch signifikant) und im Rhema $(\mathrm{H})$ (ein Unterschied von $5,86 \%$, ebenfalls statistisch signifikant) vor. Der nächste Text, den wir analysiert haben, stammt aus dem Neuhochdeutschen, aus der Zeit des Barocks, aus den Jahrzehnten unmittelbar nach dem Frühneuhochdeutschen und wird unmittelbar an die sprachlichen Verhältnisse des Frühneuhochdeutschen angeknüpft. In diesem Sinne ist die Markiertheit des synthetischen Genitivs in den Texten von Abraham a Sancta Clara zu verstehen. Um 10,79\% ist der Prozent der erweiterten Genitive (A) gestiegen (der Vergleich ist statistisch signifikant). Höher sind auch die Durchschnittszahlen der Attribute im Genitivattribut (B), im Kern (D) und die der flektierbaren Wörter (I). Der Anteil der Komparative und Superlative (E) ist höher $(5,71 \%)$ und statistisch signifikant. Die geschwächte Variante kommt auch häufiger im Rhema $(\mathrm{H})$ vor (um 1,47\%, keine statistische Signifikanz). Der Text aus dem 19. Jahrhundert nimmt hinsichtlich der gewonnenen Prozentwerte und Durchschnittszahlen eine Mittellage zwischen den Texten aus den beiden vorherigen Perioden ein. Nur bei drei Parametern haben sich allerdings Unterschiede zugunsten unserer Annahme gezeigt: die Durchschnittszahl der Attribute im Kern (D) ist höher, um 4,74 ist der Anteil der Belege ohne pronominale Elemente (F) gestiegen (keine statistische Signifikanz) und der synthetische Genitiv kommt häufiger als Akkusativobjekt vor (der Unterschied beträgt $1 \%$ und ist nicht statistisch signifikant).

Wenn wir die einzelnen Vergleiche zusammenfassen, dann können wir von einer besonderen Markiertheit der Texte aus der Barockzeit sprechen (wegen der Länge dieser schien es angebracht, zwei Texte zu analysieren). Belege aus diesen zwei Texten zeigen die höchsten Markiertheitswerte. Bei einer genaueren Einteilung aller analysierten Texte hinsichtlich der Textsorte stellt sich heraus, daß Althochdeutscher Isidor und die Texte von Meister Eckhart, Martin Luther und Matthias Scheeben eine mehr oder weniger einheitliche Gruppe bilden; es sind längere oder kürzere theologische Schriften, während Abrahams Texte aus dem Barock eine Predigt und ein Traktat sind. Der Autor gebraucht mehr Komparative und Superlative, durchschnittlich mehr flektierbare Wörter, mehr Attribute in beiden Teilen der Nominalgruppe (verglichen mit anderen Texten). Das ist typisch für die Sprache der Barockzeit (Vergleiche, Metapher, viele Attribute, ...)

Das Frühneuhochdeutsche bringt die größten Unterschiede hinsichtlich der Verbreitung des synthetischen Genitivs unter weniger einfachen Verhältnissen mit sich. Für diese Zeit vermuten wir auch die Verbreitung des analytischen Genitivs unter relativ weniger komplizierten grammatischen Verhältnissen, was aber wegen der geringen Vorkommenshäufigkeit dieser Struktur in den ausgewählten Texten nicht nachgewiesen werden konnte. In den Texten aus der Barockzeit wird diese Tendenz fortgesetzt. Einige Parameterwerte der Umgebung des synthetischen Genitivs sind zwar auch im Text aus dem 19. Jahrhundert höher, doch eine ausgeprägte Tendenz zur Verbreitung in einer weniger einfachen grammatischen Umgebung kann nicht 
beobachtet bzw. nachgewiesen werden. Eine solche auageprägte Tendenz würde bedeuten, daß die Mehrheit oder alle Parameter höhere Prozentwerte oder Durchschnittszahlen markierter Parameterwerte aufweisen.

\section{Zusammenfassung}

Bei der Analyse des synthetischen und analytischen Genitivs im Deutschen sind wir davon ausgegangen, daß verstärkte Konstruktionen vor allem in der Anfangsphase ihres Durchsetzungsprozesses bzw. Entwicklung in relativ komplizierter Umgebung, geschwächte dagegen in relativ einfacher Umgebung vorkommen.

Der analytische Genitiv kommt in den von uns analysierten Texten aus den älteren sprachgeschichtlichen Perioden der deutschen Sprache relativ selten vor. Die Vorkommenshäufigkeit der geschwächten Variante, d.h. des synthetischen Genitivs ist in allen analysierten Texten höher als die Vorkommenshäufigkeit der verstärkten Variante, d.h. des analytischen Genitivs. Der analytische Genitiv tritt auch im Text aus dem 19. Jahrhundert mit markierteren Parameterwerten des unmittelbaren grammatischen Umfeldes auf. Andererseits zeigen sich aber auch Veränderungen der grammatischen Umgebung des synthetischen Genitivs. Die grammatische Umgebung der geschwächten Konstruktion zeigt auch schon einen höheren Anteil markierter Parameterwerte im Frühneuhochdeutschen und am Anfang des Neuhochdeutschen. Eine weniger markierte Umgebung wurde im Text aus dem 19. Jahrhundert festgestellt.

Beinahe alle aufgestellten Vorhersagen haben sich durch die Analyse der Belege bestätigt. Eine Ausnahme ist die Vorhersage, daß sich verstärkte Konstruktionen später in ihrer Entwicklung in weniger markierter grammatischer Umgebung durchsetzen, die nicht überprüft werden konnte, weil ein Vergleich wegen der Zahl der Belege nicht statistisch signifikant wäre. Doch auch die Zahl der Belege bzw. die Vorkommenshäufigkeit des analytischen Genitivs spricht dafür, daß die Konstruktion eine verstärkte Variante ist.

\section{Texte}

Abraham a Sancta Clara. (1993). Ein Karren voller Narren und andere kleine Werke. Salzburg: Residenz Verlag.

Der althochdeutsche Isidor nach der Pariser Handschrift und den Monseer Fragmenten. (1964). Eggers, H. (Hsg.). Tübingen: Max Niemeyer.

Meister Eckhart. (1993). Werke II. Frankfurt am Main: Deutscher Klassiker Verlag.

Luther, M. (1982). Werke in zwei Bänden. Berlin: Aufbau-Verlag.

Scheeben, M.J. (1911). Die Mysterien des Christentums. Freiburg: Herder Verlagsbuchhandlung. 


\section{Literatur}

Admoni, W. (1985). Syntax des Neuhochdeutschen seit dem 17. Jahrhundert. In: Sprachgeschichte. Ein Handbuch zur Geschichte der deutschen Sprache und ihrer Erforschung. Besch, W., Reichmann, O., Sonderegger, S. (Hsg.). Berlin: Walter de Gruyter.

Behaghel, O. (1923). Deutsche Syntax. Band 1, Band 2, Band 3, Band 4. Heidelberg: Carl Winter.

Boretzky, N. (1977). Einführung in die historische Linguistik. Reinbek/Hamburg: Rowohlt.

Dal, I. (1966). Kurze deutsche Syntax auf historischer Grundlage. Tübingen: Max Niemeyer.

Dal, I. (1971). Untersuchungen zur germanischen und deutschen Sprachgeschichte. Oslo: Universitetsforlaget.

Leiss, E. (1991). Grammatische Kategorien und sprachlicher Wandel: Erklärung des Genitivschwunds im Deutschen. In: Proceedings of the Fourteenth International Congress of Linguistics. Berlin 10.-15.8.1987. Berlin.

Lipavic Oštir, A. (1997). Primerjava predložnega in brezpredložnega rodilnika $v$ nemščini - diahroni vidik. Magistrsko delo. Maribor.

Lipavic Oštir, A. (1997). Synthetische und analytische Formen des Genitivs im Deutschen. Manuskript anläßlich der 30.Tagung der Societas Linguistica Europaea in Göteborg, Schweden. (20.-24. August 1997).

Mayerthaler, W. (1981). Morphologische Natürlichkeit. Wiesbaden: Athenaion.

Orešnik, J. (1990). Introduction to the subsequent three papers in the present volume. In: Linguistica XXX. Ljubljana: Filozofska fakulteta.

Orešnik, J. (1995). Syntaktischer Wandel und Natürlichkeit in der Forschung slowenischer Linguisten. In: Natürlichkeitstheorie und Sprachwandel. Teorija naravnosti in jezikovno spreminjanje. Beiträge zum internationalen Symposium über "Natürlichkeitstheorie und Sprachwandel" an der Universität Maribor vom 13.5.-15.5.1993. Bochum: Universitätsverlag Brockmeyer.

Petrič, T. (1990). Posamostaljenja v knjižni nemščini. Magistrsko delo. Ljubljana.

Teržan, K. (1990). Prihodnjik kot skladenjska okrepitev - diahroni in sinhroni vidiki. Doktorska disertacija. Ljubljana.

Teržan-Kopecky, K. (1993). Analytische und synthetische Konstruktionen als morphosyntaktische Ausdrucksmittel für das Zukünftige. In: Vestnik 1-2. Ljubljana: Društvo za tuje jezike in književnosti.

\section{Povzetek}

ANALITIČNI IN SINTETIČNI RODILNIK V NEMŠČINI - DIAHRONA PRIMERJAVA

Tema prispevka je jezikovna analiza samostalniške besedne zveze kot prilastkovega rodilnika (brezpredložni ali sintetični rodilnik) in predložne zveze s predlogom von (predložni ali analitični rodilnik) v zgodovini nemškega jezika. Za jezikovno gradivo smo izbrali verske spise iz vseh obdobij razvoja jezika: Der Althochdeutsche Isidor, Meister Eckhart, Martin Luther, Abraham a Sancta Clara, Martin Scheeben. 
Teoretična izhodišča smo povzeli po slovenskem modelu naravne skladnje, ki predpostavlja soobstoj dvojice krepka in šibka zgradba, opisuje njune lastnosti, odnos med njima in njuno slovnično okolje, ki je lahko spremenljivo glede na različne faze gramatikalizacije.

Predložni rodilnik ima naslednje lastnosti krepke zgradbe: njegova glasovna in oblikovna zgradba je zapletenejša od glasovne in oblikovne podobe brezpredložnega rodilnika, pomensko je ta zgradba bolj specializirana oziroma manj splošna od brezpredložnega rodilnika (kot nadomestna zgradba se ne uporablja za vse vrste sintetičnega rodilnika), $z$ vidika govorca se predložni rodilnik teže proizvaja in $z$ vidika naslovnika se laže dekodira. Kot lastnost krepke zgradbe se je pokazala tudi pogostnost pojavljanja v starejših besedilih (sintetični rodilnik se pojavlja pogosteje) in primerjava slovničnega okolja obeh sopomenk v besedilu iz 19. stoletja, iz katere je razvidno, da se predložni rodilnik pojavlja v zapletenejšem slovničnem okolju kot brezpredložni.

Primerjava slovničnega okolja šibke zgradbe med posameznimi obdobji razvoja je pokazala širjenje le-te v manj preprosto slovnično okolje v mlajših obdobjih, posebno zaznamovanost je bilo zaznati pri obdelavi zgledov iz baročnih besedil.

Pogostnost pojavljanja in obdelava slovničnega okolja (na osnovi 1552 zgledov) obeh zgradb je potrdila vse predpostavke. Izjema je domneva o možnem širjenju krepke zgradbe v manj zapleteno slovnično okolje $v$ kasnejših stoletjih, ki zaradi nezadostnega števila zgledov ne bi izkazala statistične pomenljivosti. Toda že veliko manjša pogostnost pojavljanja (razmerje v 19. stoletju je 7,7:0,56 zgleda na stran v prid sintetičnemu rodilniku) priča o zaznamovanosti krepke sopomenke. 